\title{
Dapsone Form V: A Late Appearing Thermodynamic Polymorph of a Pharmaceutical
}

Doris E. Braun, Martin Vickers and Ulrich J. Griesser

\author{
Electronic Supplementary Information
}

Contents of ESI

A EXPERIMENTAL

1.

Pawley Fitting . 2

2.

Dapsone Form V . 3

3.

Schematic Vapor Pressure Temperature Curves of Polymorphs I - IV .

B. MODELLING

4. Computationally Generated of Low-Energy Anhydrate Structures.

5. Representation of the Experimental Structures

6. Packing Diagrams for Selected Hypothetical Low Energy Structures ..... 8

7.

7.1. CASTEP Lattice Energy Minimisations/Modelling of Form IV

7.2.

Form IV*.

8. Isomorphic Dehydrate

9. 


\section{A. EXPERIMENTAL}

\section{Pawley Fitting}

PXRD patterns of Form I and Form IV were recorded using an X'Pert PRO diffractometer (PANalytical, Almelo, $\mathrm{NL}$ ) equipped $\theta / \theta$ coupled goniometer in transmission geometry, programmable $\mathrm{XYZ}$ stage with well plate holder, $\mathrm{Cu}-\mathrm{K \alpha}_{1,2}$ radiation source with a focusing mirror, a $0.5^{\circ}$ divergence slit and a $0.02^{\circ}$ Soller slit collimator on the incident beam side, a $2 \mathrm{~mm}$ antiscattering slit and a $0.02^{\circ}$ Soller slit collimator on the diffracted beam side and a solid-state PIXcel detector. The patterns were recorded at a tube voltage of $40 \mathrm{kV}$ and tube current of $40 \mathrm{~mA}$, applying a step size of $2 \theta=0.013^{\circ}$ with $80 \mathrm{~s}$ per step in the $2 \theta$ range between $2^{\circ}$ and $40^{\circ}$. Pawley fits ${ }^{1}$ were performed with Topas Academic V5. ${ }^{2}$ The background was modelled with Chebyshev polynomials and the modified Thompson-Cox-Hastings pseudo-Voigt (TCHZ) function was used for peak shape fitting.

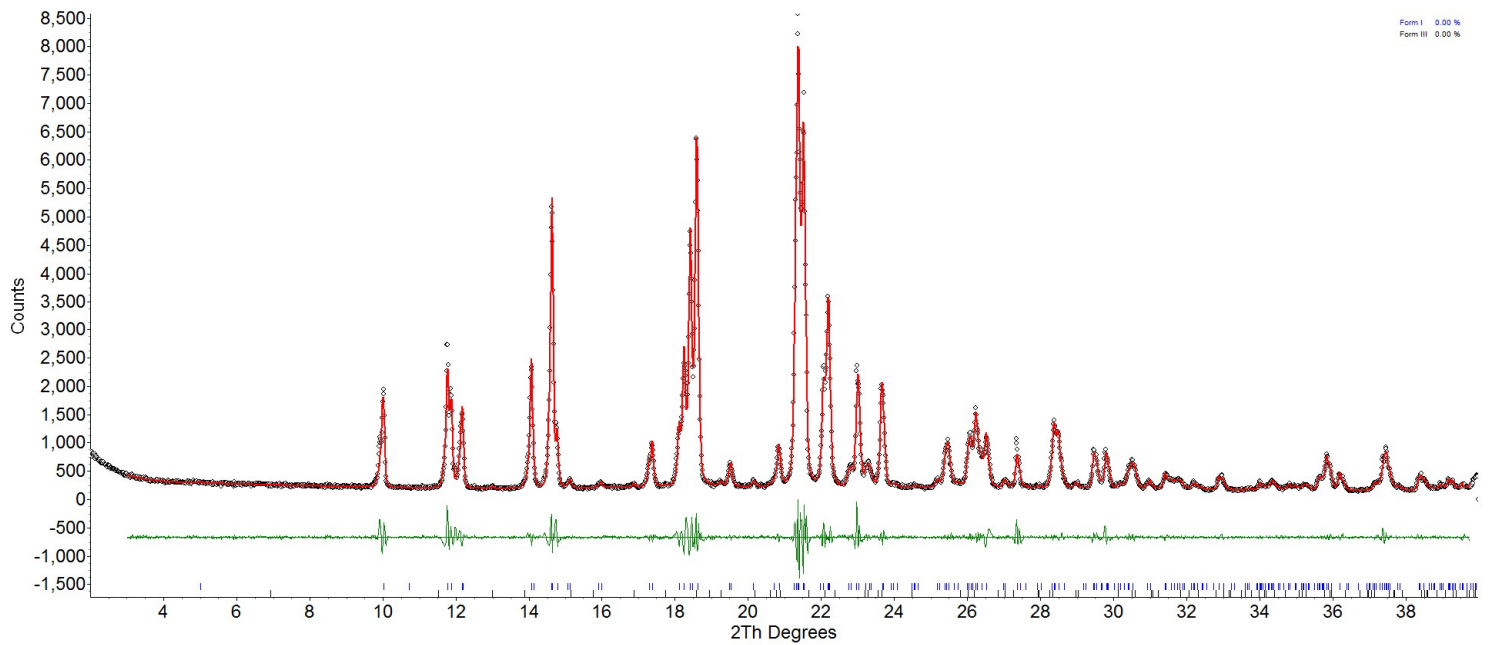

Figure S1. Pawley fit $\left(R_{\mathrm{wp}}=7.58 \%, R_{\exp }=4.27 \%, R_{\mathrm{p}}=5.62 \%\right)$ between the PXRD data of Form I with a model consisting of the cell parameters derived from indexing the PXRD pattern and Form III (impurity). Black dots indicate the raw data, while the red line indicates the calculated model. Tick marks (blue - Form I, black - Form III) are the $2 \theta$ positions for the $h k l$ reflections. The difference pattern is shown in green.

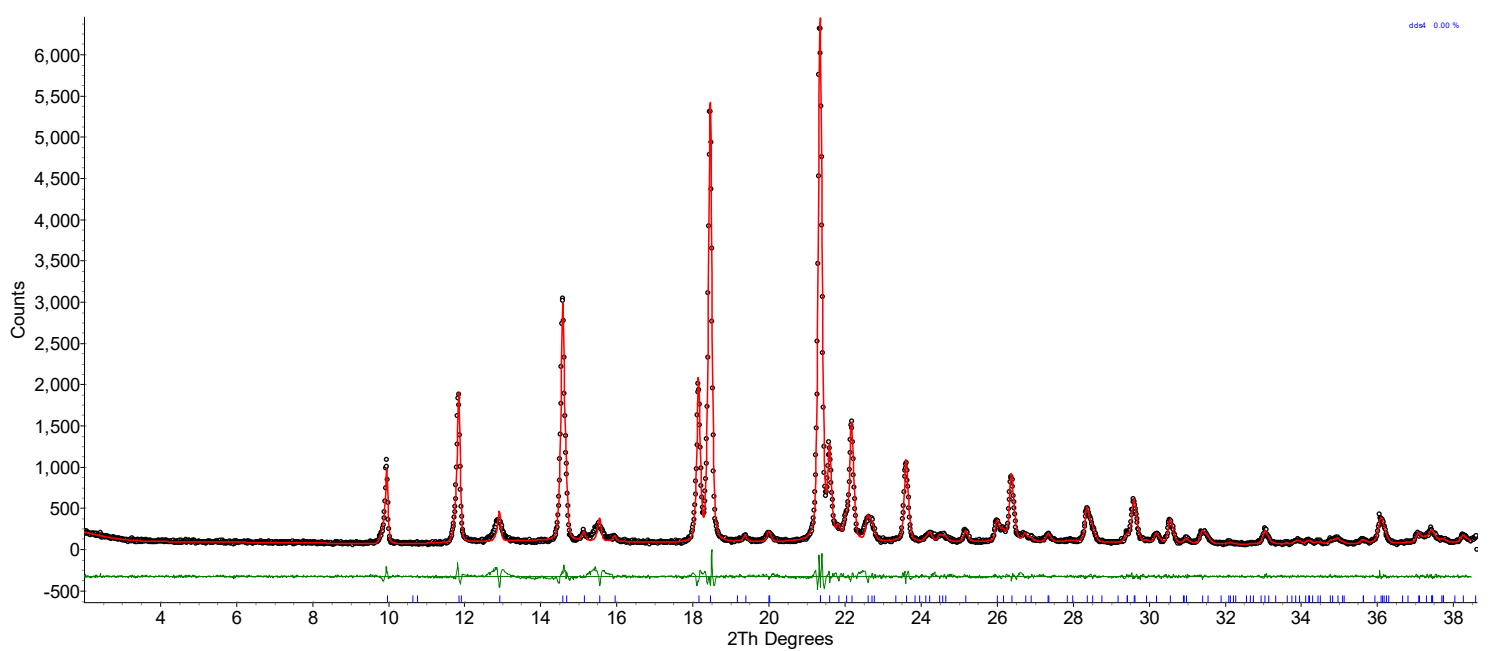

Figure S2. Pawley fit $\left(R_{\mathrm{wp}}=9.43 \%, R_{\exp }=6.73 \%, R_{\mathrm{p}}=6.50 \%\right)$ between the PXRD data of Form IV with a model consisting of the cell parameters derived from indexing the PXRD pattern. Black dots indicate the raw data, while the red line indicates the calculated model. Blue tick marks are the $2 \theta$ positions for the $h k l$ reflections. The difference pattern is shown in green. 


\section{Dapsone Form V}

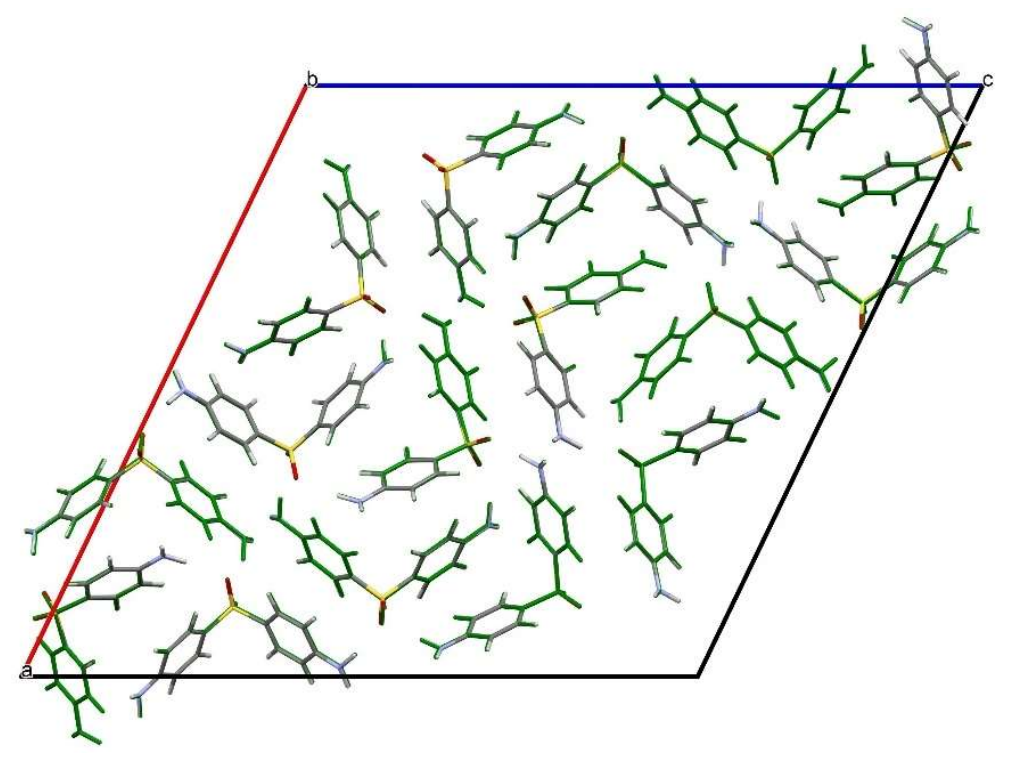

Figure S3. Overlay of the 30 molecule cluster of DDS Form $\mathbf{V}$ (colored by element) and calculated PBE-TS with lattice parameters fixed to experimental values (green), $r m s d_{30}=0.04 \AA$. Viewed along the crystallographic $b$ axes.

\section{Schematic Vapor Pressure Temperature Curves of Polymorphs I - IV}

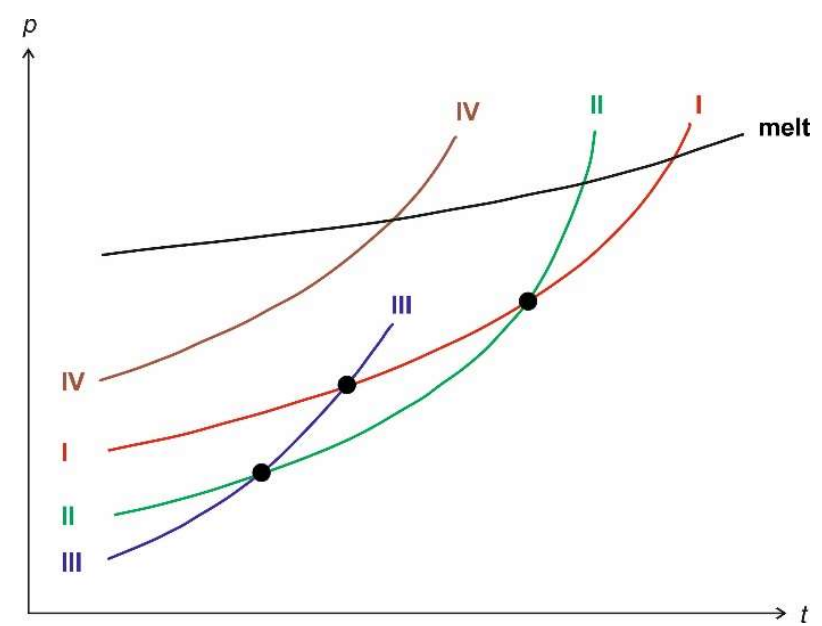

Figure S4. Vapor-pressure temperature curves of DDS Forms I - IV and the melt. Redrawn from ref. 3. 


\section{B. MODELLING}

\section{Computationally Generated of Low-Energy Anhydrate Structures}

The lowest energy PBE-D2 structures are given in Table S1.

Table S1. Known and computationally generated low energy (PBE-TS optimized and PBE-D2 single point calculations) structures. The experimental structures are highlighted in grey.

\begin{tabular}{|c|c|c|c|c|c|c|c|c|c|c|}
\hline \multirow[t]{2}{*}{ Str. ${ }^{a}$} & \multirow{2}{*}{$\begin{array}{c}\text { Space group } \\
\left(Z^{\prime}\right)\end{array}$} & \multicolumn{6}{|c|}{ Cell parameters } & \multirow{2}{*}{$\begin{array}{c}\mathrm{E}_{\text {latt }} / \\
\mathrm{kJ} \mathrm{mol}^{-1}\end{array}$} & \multirow{2}{*}{$\begin{array}{c}\mathrm{PI}^{\mathrm{b}} / \\
\% \\
\end{array}$} & \multirow{2}{*}{$\begin{array}{l}\text { Void } \\
\text { space }^{c}\end{array}$} \\
\hline & & $a / \AA ̊$ & $b / \AA$ & $c / \AA$ & $\alpha /^{\circ}$ & $8 /^{\circ}$ & $\gamma /{ }^{\circ}$ & & & \\
\hline 01_V & $P 2_{1} / n(4)$ & 29.186 & 5.698 & 30.126 & 90 & 115.53 & 90 & -198.53 & 73.8 & 0 \\
\hline 02_19 (III) & $P 2_{1} 2_{1} 2_{1}(1)$ & 5.498 & 7.975 & 26.050 & 90 & 90 & 90 & -198.21 & 72.9 & 0 \\
\hline 03_1311 & $P_{c a 2_{1}}(2)$ & 15.669 & 5.674 & 25.827 & 90 & 90 & 90 & -197.86 & 72.4 & 1.7 \\
\hline $04 \_21$ & $\operatorname{Pna2}_{1}$ (1) & 5.584 & 26.874 & 7.866 & 90 & 90 & 90 & -197.34 & 70.7 & 6.3 \\
\hline 05_dehy & $C 2 / c(3)$ & 48.216 & 11.347 & 12.950 & 90 & 92.64 & 90 & -196.72 & 70.4 & 3.7 \\
\hline 06_II & $P 2_{1} 2_{1} 2_{1}(1)$ & 5.770 & 7.902 & 25.061 & 90 & 90 & 90 & -195.09 & 73.3 & 0 \\
\hline 07_591 & $\mathrm{Pca}_{1}(2)$ & 15.538 & 5.836 & 25.670 & 90 & 90 & 90 & -195.05 & 71.6 & 2.1 \\
\hline $08 \_800(\mathrm{I})$ & $P 2_{1} / c(2)$ & 18.979 & 8.282 & 16.390 & 90 & 114.60 & 90 & -194.61 & 71.2 & 0 \\
\hline 09_3410 & $P 2_{1} / c(2)$ & 27.301 & 8.169 & 10.710 & 90 & 100.10 & 90 & -194.59 & 71.1 & 4.5 \\
\hline 10_155 & $\operatorname{Pna}_{1}(2)$ & 14.100 & 26.973 & 6.064 & 90 & 90 & 90 & -194.34 & 72.4 & 0 \\
\hline 11_4478 & $P 2_{1} / c(1)$ & 14.637 & 5.709 & 15.350 & 90 & 117.34 & 90 & -194.27 & 73.2 & 1.9 \\
\hline 12_322 & $P 2_{1}(2)$ & 5.633 & 7.975 & \begin{tabular}{|l|}
25.702 \\
\end{tabular} & 90 & 90.84 & 90 & -192.80 & 72.5 & 0 \\
\hline 13_2871 & $P 2_{1}(2)$ & 5.552 & 7.833 & \begin{tabular}{|l|}
27.244 \\
\end{tabular} & 90 & 90.37 & 90 & -192.73 & 70.4 & 6.6 \\
\hline 14_409 & $P 2_{1} / c(2)$ & 5.829 & 28.053 & 14.002 & 90 & 93.70 & 90 & -192.02 & 73.1 & 0 \\
\hline 15_944 & Pbca (1) & 5.702 & 15.436 & 26.545 & 90 & 90 & 90 & -191.68 & 71.4 & 0.8 \\
\hline $16 \_1772$ & $P 2_{1} / c(2)$ & 26.842 & 8.160 & \begin{tabular}{|l|}
10.734 \\
\end{tabular} & 90 & 100.77 & 90 & -191.16 & 72.3 & 0 \\
\hline 17_1389 & $P 2_{1} / c(1)$ & 7.962 & 13.466 & \begin{tabular}{|l|}
10.412 \\
\end{tabular} & 90 & 92.27 & 90 & -191.10 & 74.9 & 0 \\
\hline $18 \_2291$ & $P_{n a 2_{1}}(2)$ & 15.030 & 5.647 & \begin{tabular}{|l|}
26.284 \\
\end{tabular} & 90 & 90 & 90 & -191.03 & 74.8 & 0 \\
\hline 19_6694 & Pbca (2) & 15.780 & 16.236 & 19.030 & 90 & 90 & 90 & -190.87 & 68.1 & 2.2 \\
\hline 20_987 & $P 2_{1} / c(2)$ & 27.133 & 5.624 & 15.108 & 90 & 104.05 & 90 & -190.72 & 74.6 & 0 \\
\hline 21_69 & $P 2_{1} / c(2)$ & 26.754 & 5.784 & 15.240 & 90 & 105.92 & 90 & -190.54 & 73.7 & 0 \\
\hline 22_1063 & $P 2_{1} / n(2)$ & 15.205 & 7.969 & 19.069 & 90 & 98.63 & 90 & -190.09 & 73.2 & 0 \\
\hline 23_5504 & $P c a 2_{1}(2)$ & 29.001 & 5.713 & 14.013 & 90 & 90 & 90 & -190.00 & 71.9 & 1.9 \\
\hline 24_697 (VI) & $P c a 2_{1}(2)$ & 16.264 & 8.291 & \begin{tabular}{|l|}
17.709 \\
\end{tabular} & 90 & 90 & 90 & -189.70 & 70 & 0 \\
\hline 25_188 & $P 2_{1} / c(2)$ & 5.957 & 29.689 & \begin{tabular}{|l|}
13.136 \\
\end{tabular} & 90 & 96.41 & 90 & -189.65 & 72.5 & 0 \\
\hline 26_198 & $\mathrm{Pca}_{1}(2)$ & 5.816 & 26.672 & 14.980 & 90 & 90 & 90 & -189.53 & 71.9 & 1.4 \\
\hline $27 \_2423$ & $P 2_{1} / c(2)$ & 8.412 & 15.112 & 18.865 & 90 & 98.80 & 90 & -189.41 & 70.4 & 2.2 \\
\hline 28_1606 & la (2) & 15.004 & 5.673 & 27.792 & 90 & 103.10 & 90 & -189.18 & 72.6 & 1.1 \\
\hline 29_58 & $\operatorname{Pna}_{1}(2)$ & 15.344 & 5.937 & 25.401 & 90 & 90 & 90 & -189.16 & 72.2 & 0 \\
\hline 30_5243 & $P 2_{1}(2)$ & 5.687 & 26.333 & 8.203 & 90 & 106.50 & 90 & -189.15 & 70.8 & 2.9 \\
\hline 31_1297 & $P 21 / c(2)$ & 25.606 & 5.765 & 15.307 & 90 & 90.78 & 90 & -189.03 & 74 & 0 \\
\hline $32 \_5476$ & $C 2(2)$ & 15.623 & 5.745 & \begin{tabular}{|l|}
27.230 \\
\end{tabular} & 90 & 104.72 & 90 & -188.88 & 70.6 & 3 \\
\hline 33_196 & Ic (2) & 14.964 & 5.843 & \begin{tabular}{|l|}
27.405 \\
\end{tabular} & 90 & 104.10 & 90 & -188.83 & 72 & 1.9 \\
\hline 34_180 & $P 2_{1} / c(1)$ & 5.854 & 7.812 & \begin{tabular}{|l|}
25.160 \\
\end{tabular} & 90 & 93.14 & 90 & -188.81 & 72.7 & 0 \\
\hline 35_148 & $P 2_{1} 2_{1} 2_{1}(2)$ & 7.510 & 13.239 & 23.044 & 90 & 90 & 90 & -188.79 & 72.9 & 1.3 \\
\hline 36_142 & $P 2_{1} / n(2)$ & 13.294 & 7.423 & \begin{tabular}{|l|}
24.031 \\
\end{tabular} & 90 & 105.57 & 90 & -188.70 & 73.1 & 1.1 \\
\hline 37_75 & $P 2_{1} / c(2)$ & 25.935 & 5.856 & 15.262 & 90 & 94.99 & 90 & -188.56 & 72.5 & 0 \\
\hline $38 \_47$ & $\operatorname{Pca}_{1}(2)$ & 7.893 & 11.914 & 24.241 & 90 & 90 & 90 & -188.51 & 73.5 & 0 \\
\hline 39_3009 & $P 2_{1} / c(2)$ & 14.170 & 5.991 & 15.479 & 90 & 112.02 & 90 & -188.40 & 68.5 & 5.2 \\
\hline 40_8847 (desolv) & $P 21 / n(2)$ & 8.459 & 16.312 & 19.460 & 90 & 99.705 & 90 & -188.38 & 62.9 & 15.6 \\
\hline
\end{tabular}

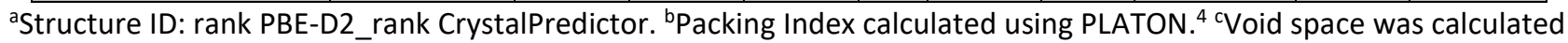
using a $1.0 \AA$ probe radius and an approx. grid spacing of $0.15 \AA$ and is given as \% of unit cell volume. 


\section{Representation of the Experimental Structures}

The computational models were successful in reproducing the experimental structures (Table S2). The structures were compared using the Molecular Similarity Module in Mercury to determine the root mean square deviation of the non-hydrogen atoms in a cluster of $n$ molecules $\left(r m s d_{n}\right) .^{5}$

Table S2. Quality of representation of the experimental and calculated (PBE-TS) anhydrate and hydrate structures of DDS.

\begin{tabular}{|c|c|c|c|c|c|c|}
\hline \multirow[b]{2}{*}{ Solid Form } & \multicolumn{4}{|c|}{ Lattice parameters (cell vectors $/ \AA ̊$, angles $/{ }^{\circ}$ ) } & \multirow{2}{*}{$\begin{array}{c}\text { cell volume } \\
\qquad\left(\AA^{3}\right)\end{array}$} & \multirow{2}{*}{$\begin{array}{c}\operatorname{rmsd}_{15 / 30} \\
(\AA)\end{array}$} \\
\hline & $a / \AA$ & $b / \AA$ & $c / \AA$ & $\beta /^{\circ}$ & & \\
\hline \multicolumn{7}{|c|}{ Anhydrates } \\
\hline Form III, DAPSUO05, RT, $P 22_{1} 2_{1} 2_{1}$ & 5.758 & 8.058 & 25.529 & 90 & 1184.49 & - \\
\hline Form III PBE-TS, $0 \mathrm{~K}$ & 5.498 & 7.975 & 26.050 & 90 & 1142.20 & 15: 0.239 \\
\hline Form II, DAPSU015, $363 \mathrm{~K}, P 2{ }_{1} 2_{1} 2_{1}$ & 5.866 & 7.990 & 25.171 & 90 & 1179.85 & - \\
\hline Form II, PBE-TS, $0 \mathrm{~K}$ & 5.770 & 7.902 & 25.061 & 90 & 1142.48 & 15: 0.206 \\
\hline Form $\mathbf{V}$, exptl, $\mathrm{RT}, P 2_{1} / c$ & 29.340 & 5.841 & 30.272 & 115.766 & 4672.19 & - \\
\hline Form V PBE-TS, $0 \mathrm{~K}$ & 29.186 & 5.698 & 30.126 & 115.527 & 4520.77 & 30: 0.140 \\
\hline \multicolumn{7}{|c|}{ Hydrate } \\
\hline 0.33-Hy, ANSFONO2, $120 \mathrm{~K}, \mathrm{C2} / \mathrm{c}$ & 48.5832 & 11.4183 & 13.0035 & 92.279 & 7207.82 & - \\
\hline 0.33-Hy, PBE-TS, O K & 48.1926 & 11.3940 & 13.0023 & 92.100 & 7134.85 & 30: 0.052 \\
\hline
\end{tabular}

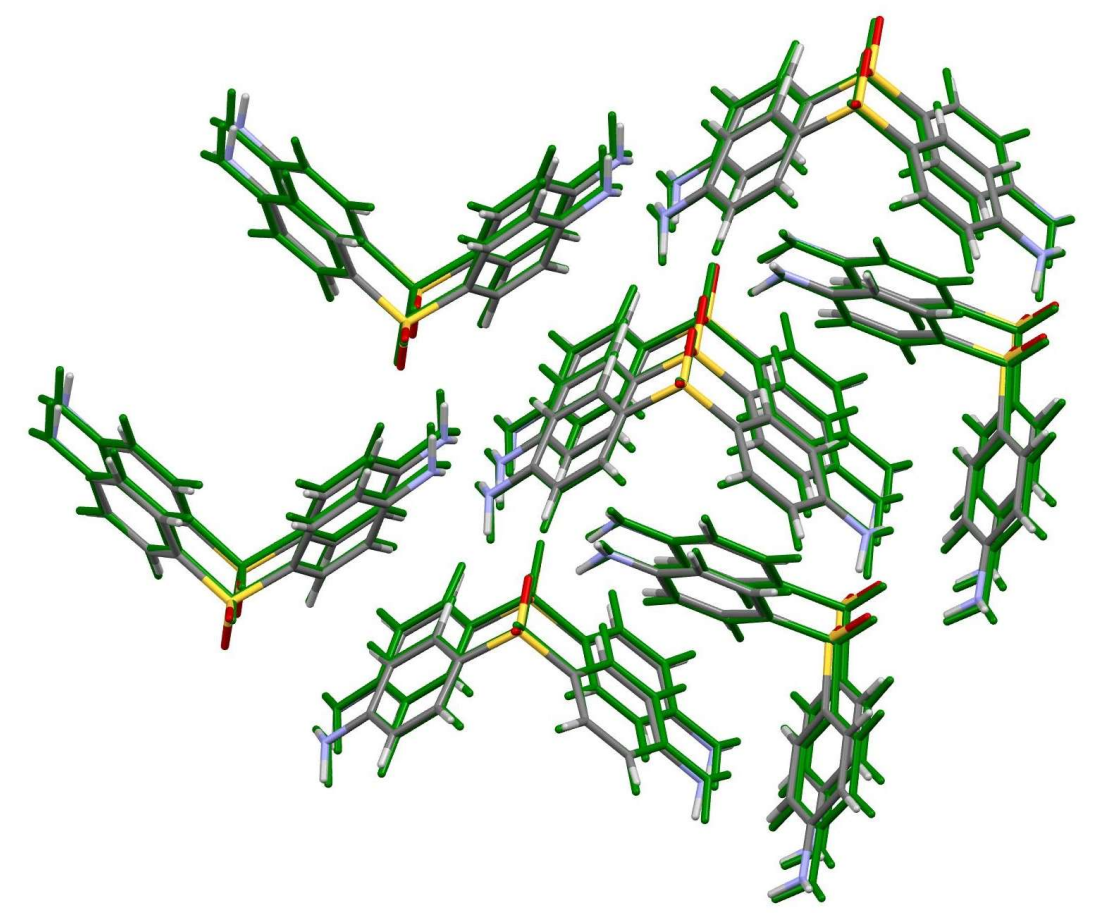

Figure S5. Overlay of the 15 molecule cluster of the observed structure of DDS Form III (colored by element) and calculated PBE-TS structure (green), $r m s d_{15}=0.24 \AA$. 


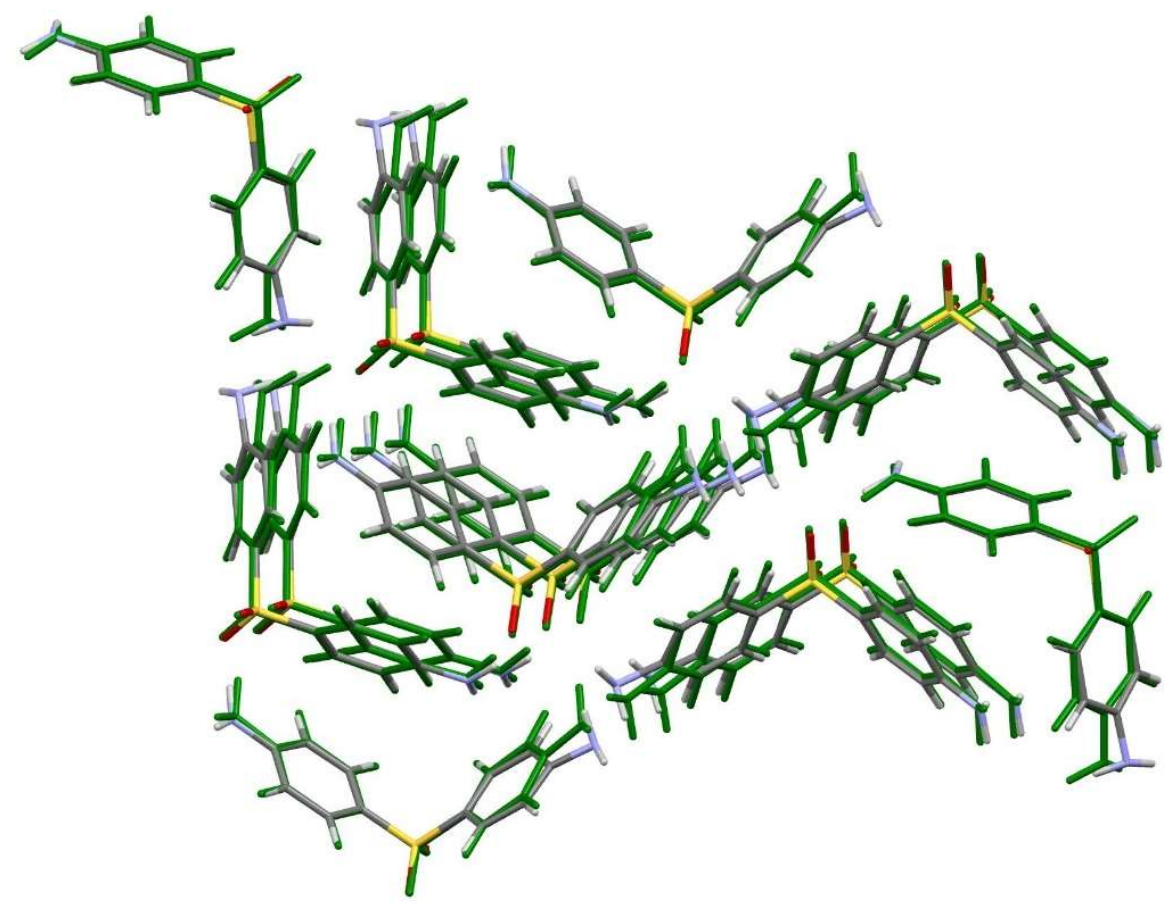

Figure S6. Overlay of the 15 molecule cluster of the observed structure of DDS Form II (colored by element) and calculated PBE-TS structure (green), $r m s d_{15}=0.21 \AA$.

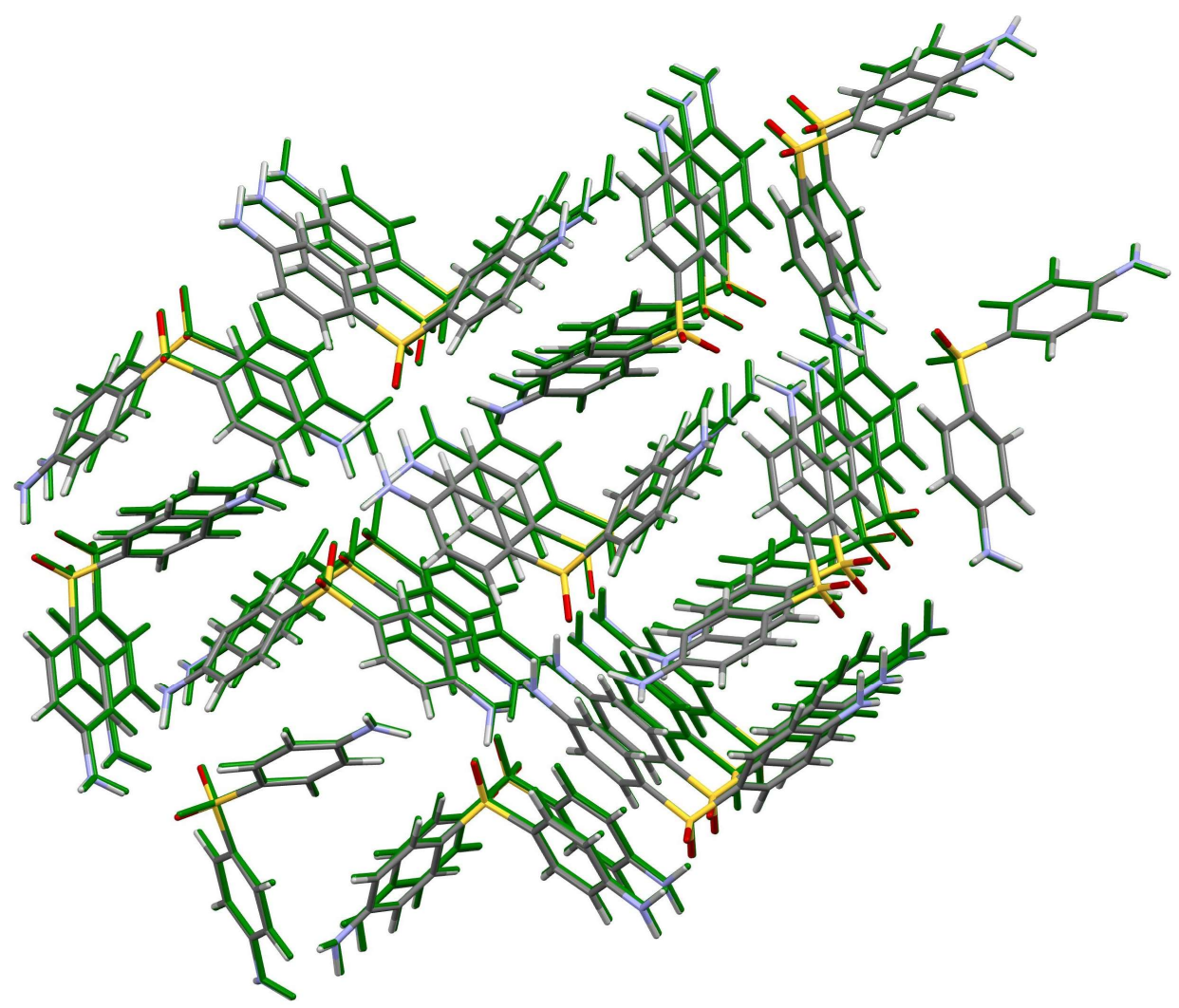

Figure S7. Overlay of the 30 molecule cluster of the observed structure of DDS Form $\mathbf{V}$ (colored by element) and calculated PBE-TS structure (green), $r m s d_{30}=0.14 \AA$. 


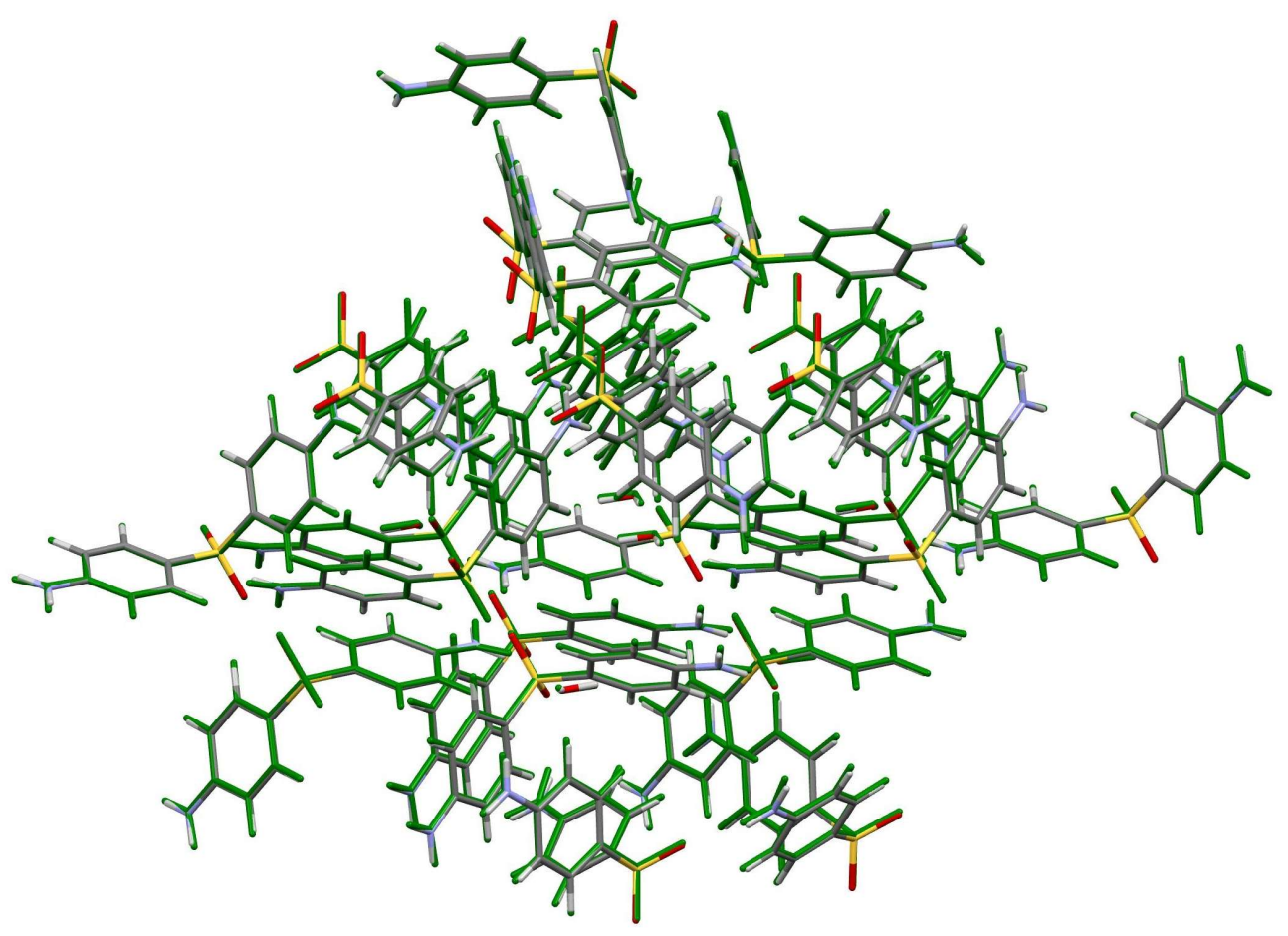

Figure S8. Overlay of the 30 molecule cluster of the observed structure of DDS $\mathbf{0 . 3 3 - H y}$ (colored by element) and calculated PBE-TS structure (green), $r m s d_{30}=0.05 \AA$. 


\section{Packing Diagrams of Selected Hypothetical Low Energy Structures}

\subsection{Rank 3}

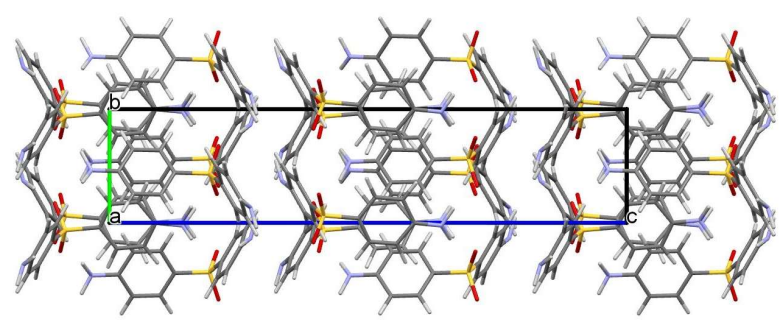

(a) viewed along $a$

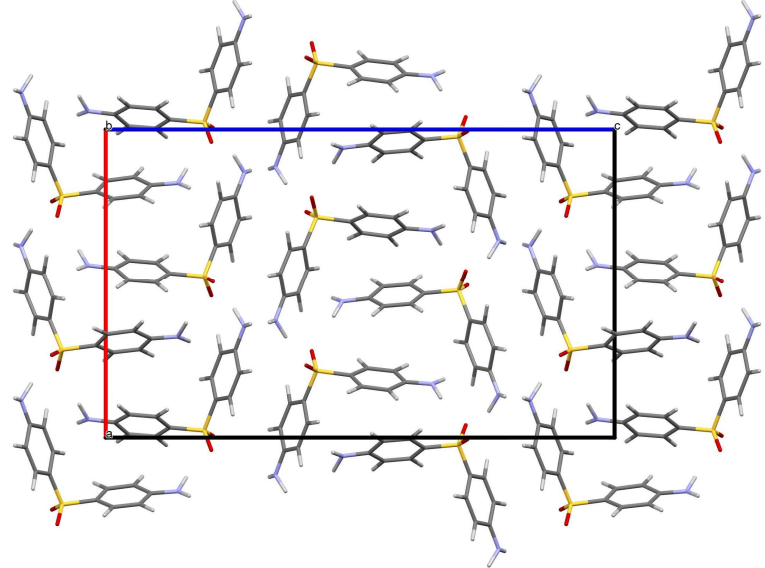

(b) viewed along $b$

Figure S9. Packing diagrams of DDS rank 3 anhydrate structure.

\subsection{Rank 4}

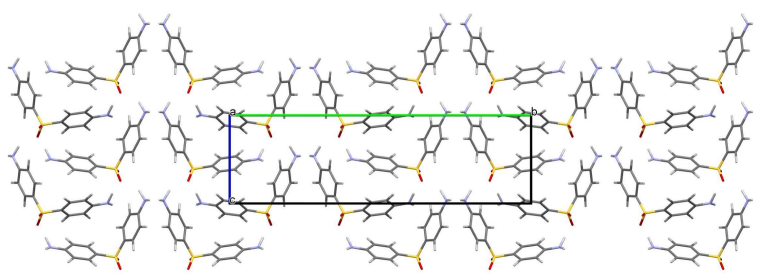

(a) viewed along $a$

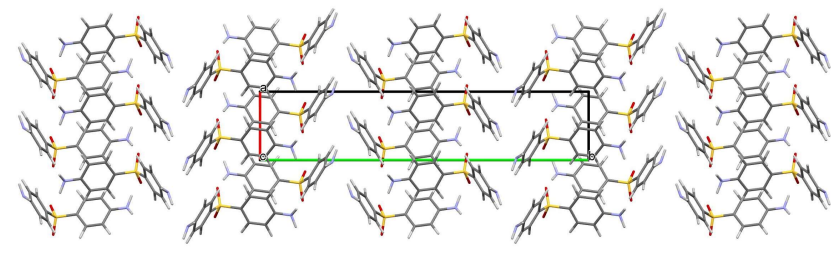

(a) viewed along $c$

Figure S10. Packing diagrams of DDS rank 4 anhydrate structure.

\subsection{Rank 7}

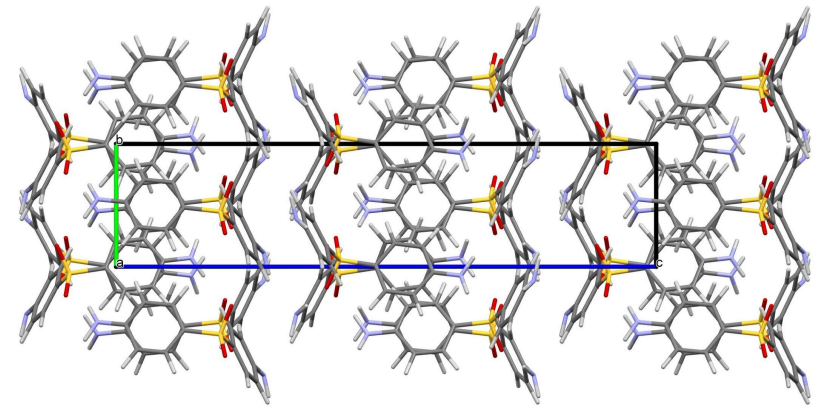

(a) viewed along $a$

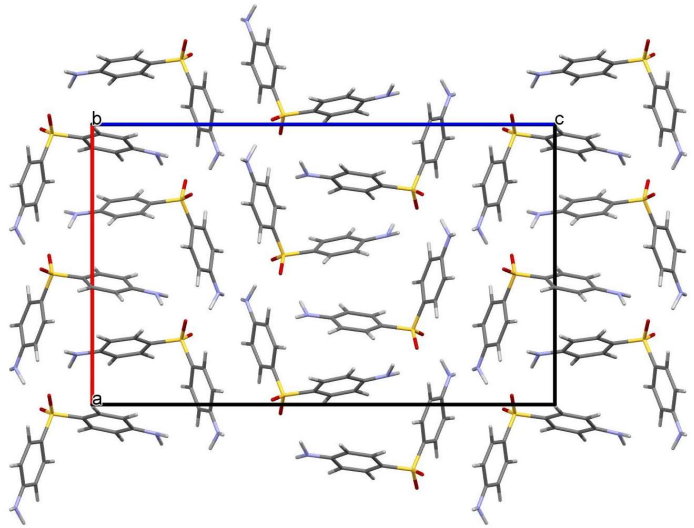

(a) viewed along $b$

Figure S11. Packing diagrams of DDS rank 7 anhydrate structure. 


\section{CASTEP Lattice Energy Minimizations/Modelling of Form IV}

\subsection{Form IV*}

Potential Form IV structure models were generated starting from the predicted rank 24 structure. The $P c a 2_{1}\left(Z^{\prime}=2\right)$ cell was transformed to a $P 1\left(Z^{\prime}=8\right)$ structure and selected DDS molecules were rotated within the $P 1$ structure. The resulting input structures were then optimized using CASTEP (Figure S12) without symmetry restraints.

(a) (b)




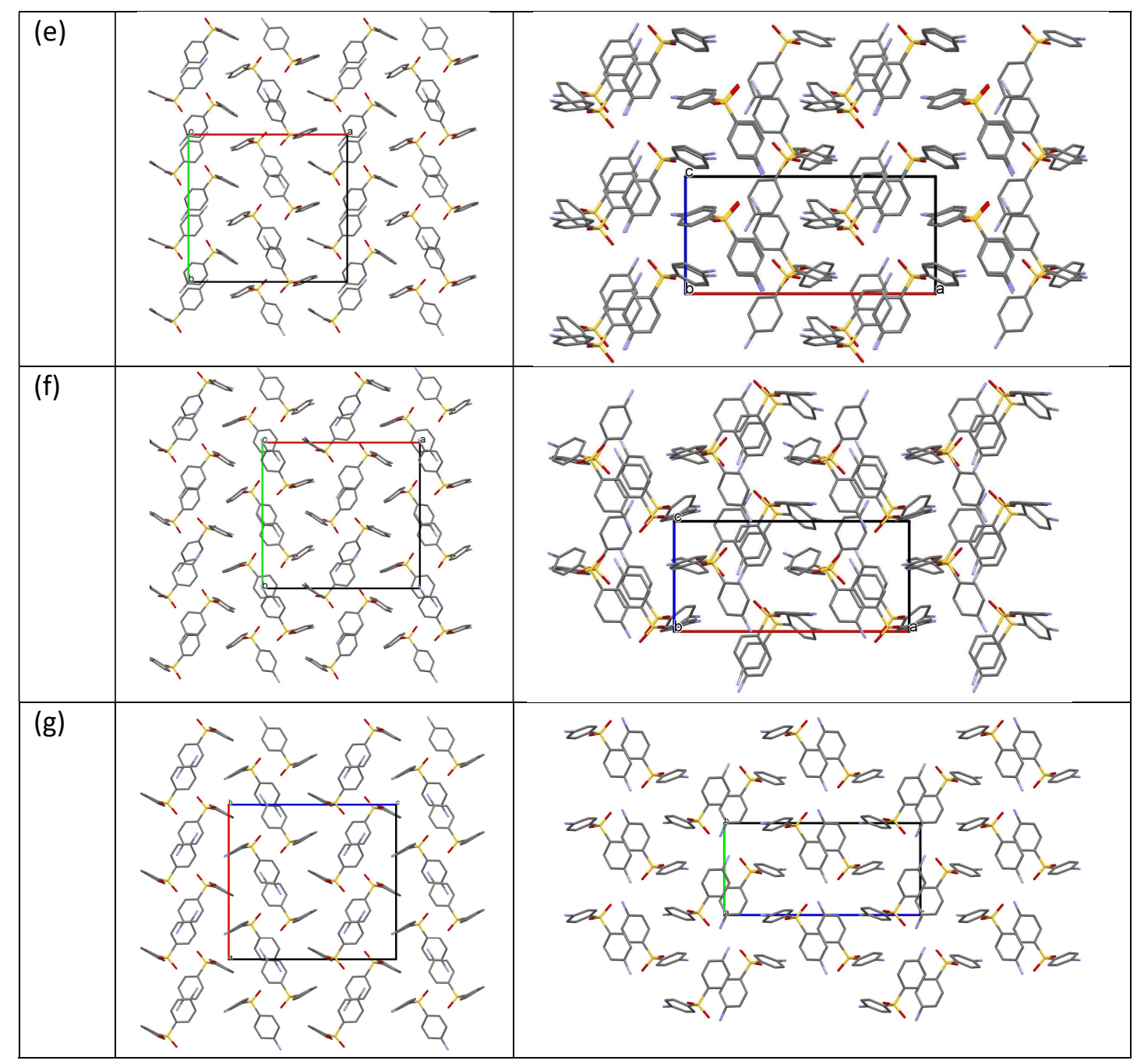

Figure S12. Disorder modelling of Form IV. Structures (a-d) correspond to the four models given in the $\mathrm{m} / \mathrm{s}$ (Figure 13). 


\subsection{Isomorphic Dehydrate}

Computational removal of the water molecules from the $\mathbf{0 . 3 3 - H y ~ s t r u c t u r e ~ a l l o w e d ~ u s ~ t o ~ d e r i v e ~ t h e ~}$

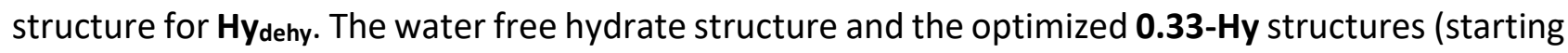
from ANSFONO2 ${ }^{6}$ and using the settings as described in the $\mathrm{m} / \mathrm{s}$ in section 2.7 ) resulted in two minima that are superimposable with the exception of the water molecules $\left[r m s d_{30}(D D S)=0.065\right.$ $\AA ̊]$.

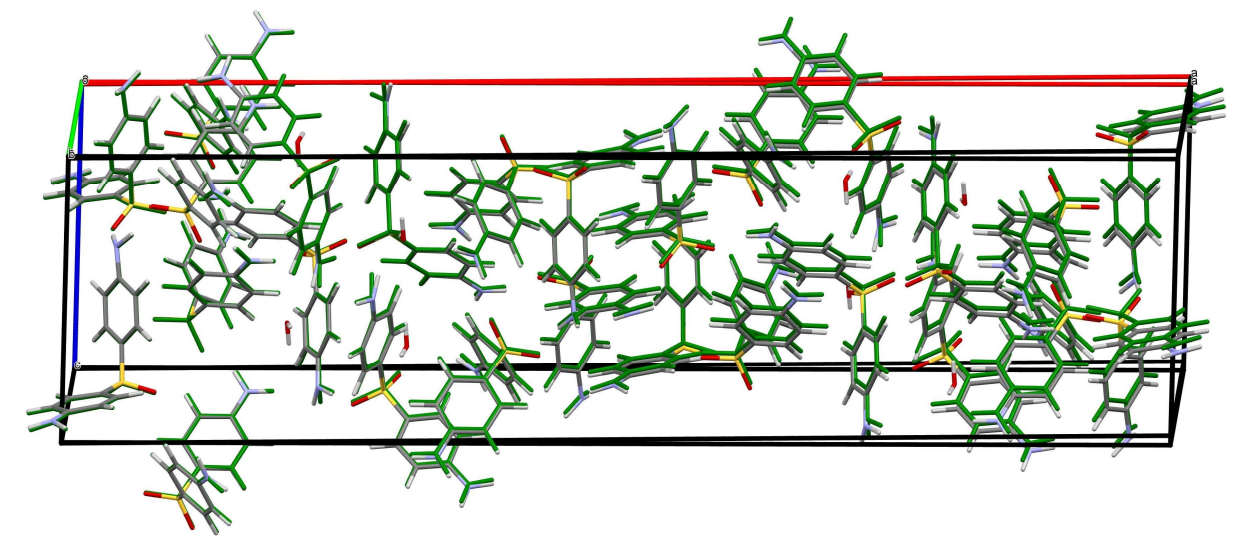

Figure S13. Overlay of the unit cell of $\mathbf{0 . 3 3 - H y}$ (in color) and Hydehy (green). 


\section{CrystalExplorer Calculations}

The pairwise energy contributions to experimental and computed DDS solid forms have been calculated using CrystalExplorer V17..$^{-9}$ The optimized atomic positions (PBE-TS) have been used in all subsequent intermolecular interaction energy calculations. The model energies have been calculated between all unique nearest neighbor molecular pairs. The used model (termed CE-B3LYP) uses B3LYP/6-31G(d,p) molecular wave functions calculated by applying the molecular geometries extracted from the crystal structures. This approach uses electron densities of unperturbed monomers to obtain four separate energy components: electrostatic $\left(E_{\mathrm{E}}\right)$, polarization $\left(E_{\mathrm{P}}\right)$, dispersion $\left(E_{\mathrm{D}}\right)$, and exchange-repulsion $\left(E_{\mathrm{R}}\right)$. Each energy term was scaled independently to fit a large training set of B3LYP-D2/6-31G(d,p) counterpoise-corrected energies from both organic and inorganic crystals. The CE-B3LYP energies reproduced the training set energies with a mean absolute deviation of $\sim 1 \mathrm{~kJ} \mathrm{~mol}^{-1}$.

\subsection{1_Form V}

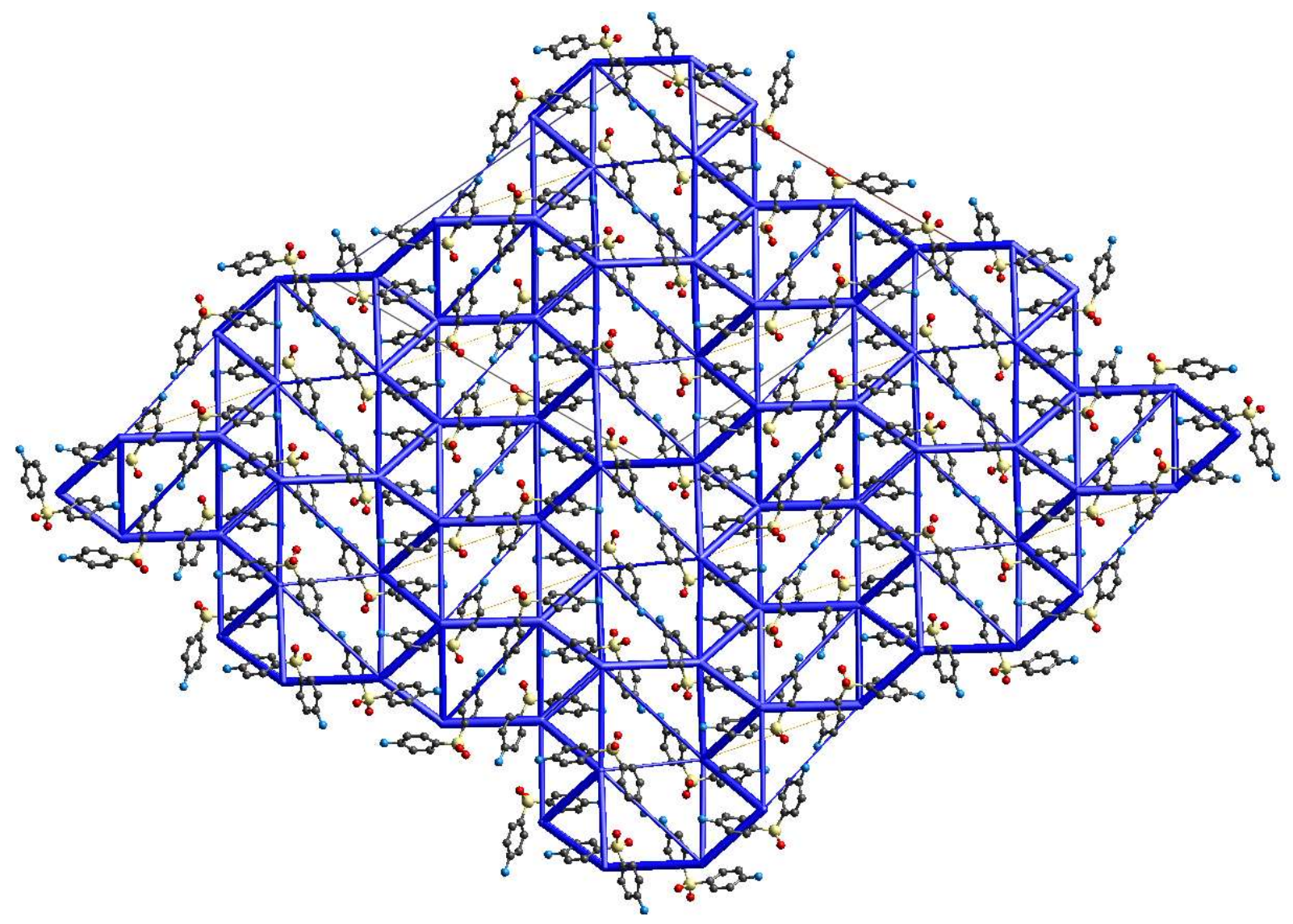

Figure S14. Energy frameworks (total energy) for Form V, viewed along the crystallographic $b$ axis. The energy scale factor is 80 , and pairwise interaction energies with magnitudes smaller than $20 \mathrm{~kJ} \mathrm{~mol}^{-1}$ have been omitted. 
8.2.

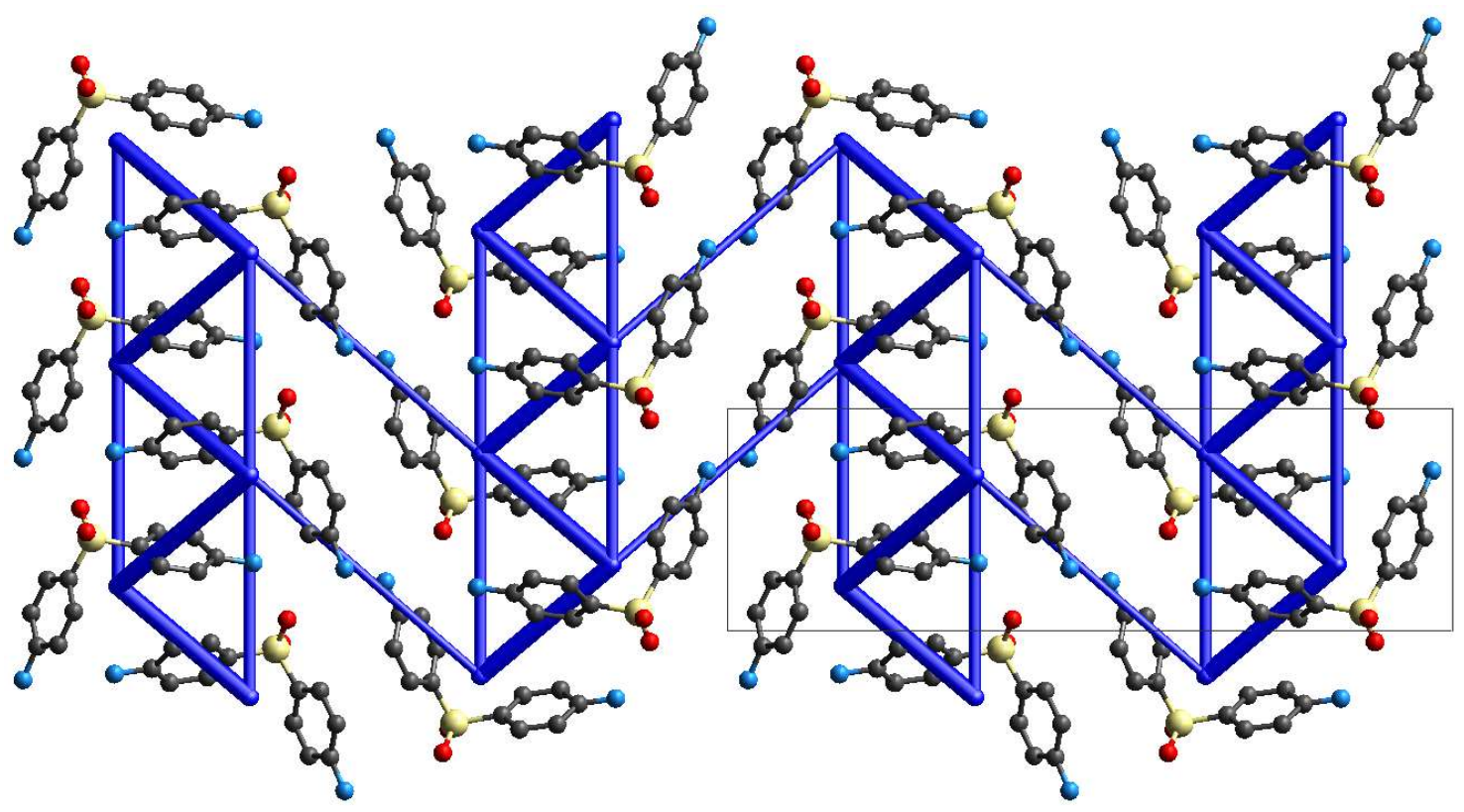

Figure S15. Energy frameworks (total energy) for Form III, viewed along the crystallographic $a$ axis. The energy scale factor is 80 , and pairwise interaction energies with magnitudes smaller than $20 \mathrm{~kJ} \mathrm{~mol}^{-1}$ have been omitted.

\subsection{3_1311}

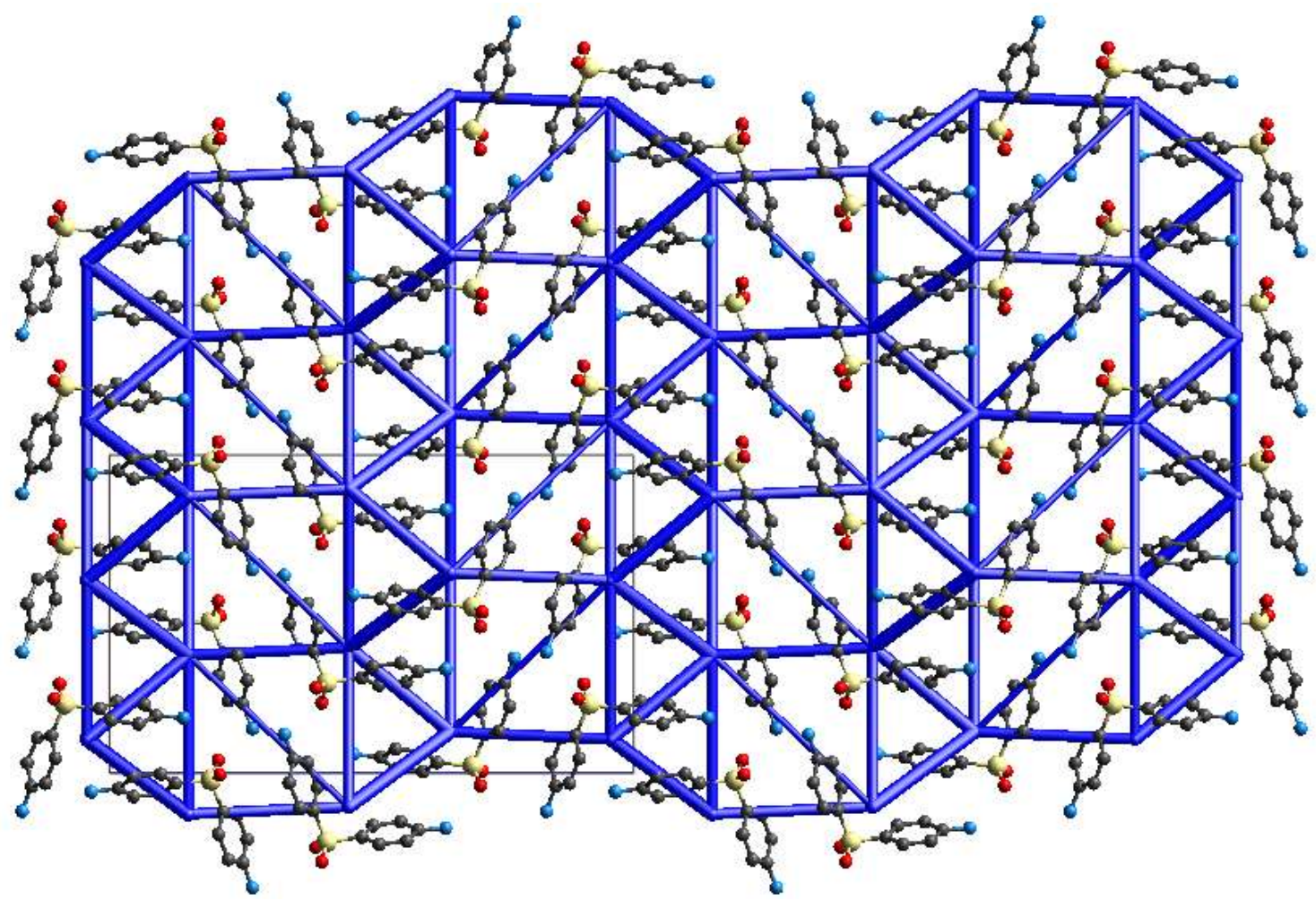

Figure S16. Energy frameworks (total energy) for 03_1311, viewed along the crystallographic $b$ axis. The energy scale factor is 80 , and pairwise interaction energies with magnitudes smaller than $20 \mathrm{~kJ} \mathrm{~mol}^{-1}$ have been omitted. 


\subsection{4_21}

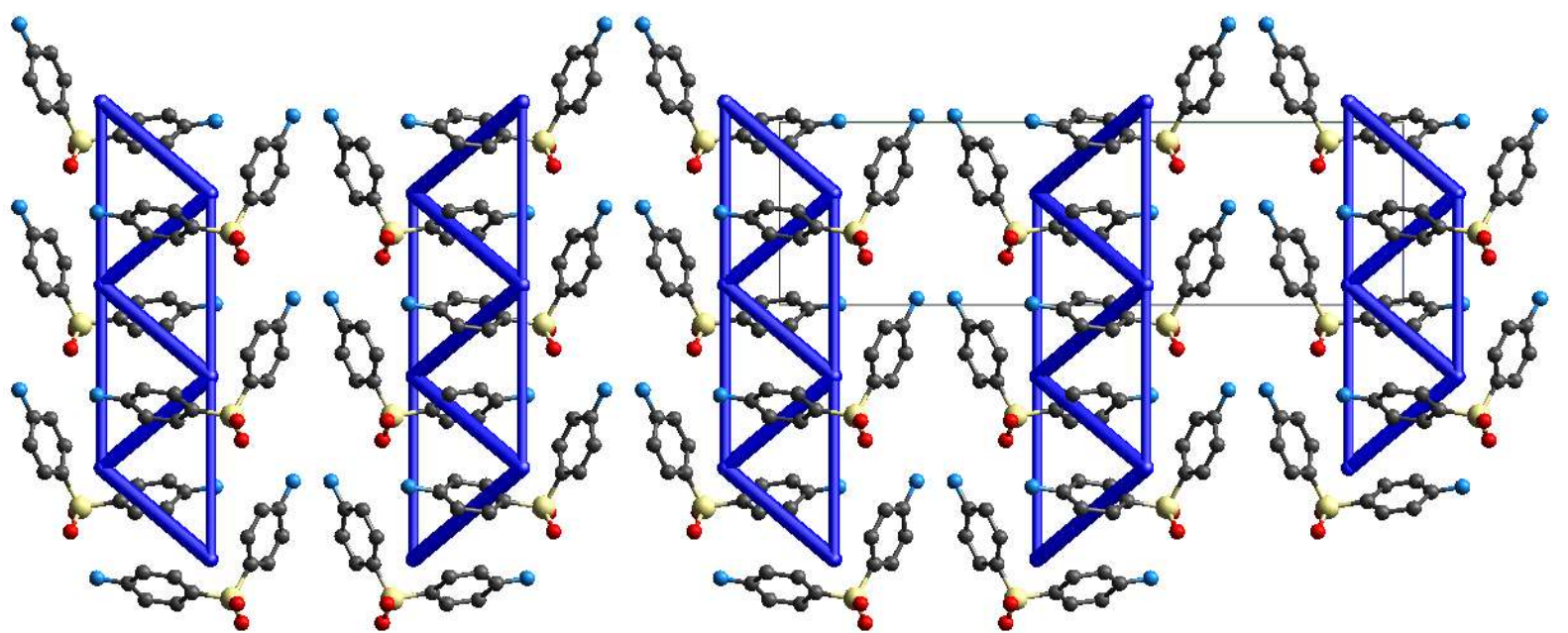

Figure S17. Energy frameworks (total energy) for 04_21, viewed along the crystallographic $a$ axis. The energy scale factor is 80 , and pairwise interaction energies with magnitudes smaller than $20 \mathrm{~kJ} \mathrm{~mol}^{-1}$ have been omitted.

\subsection{5_Hydehy}

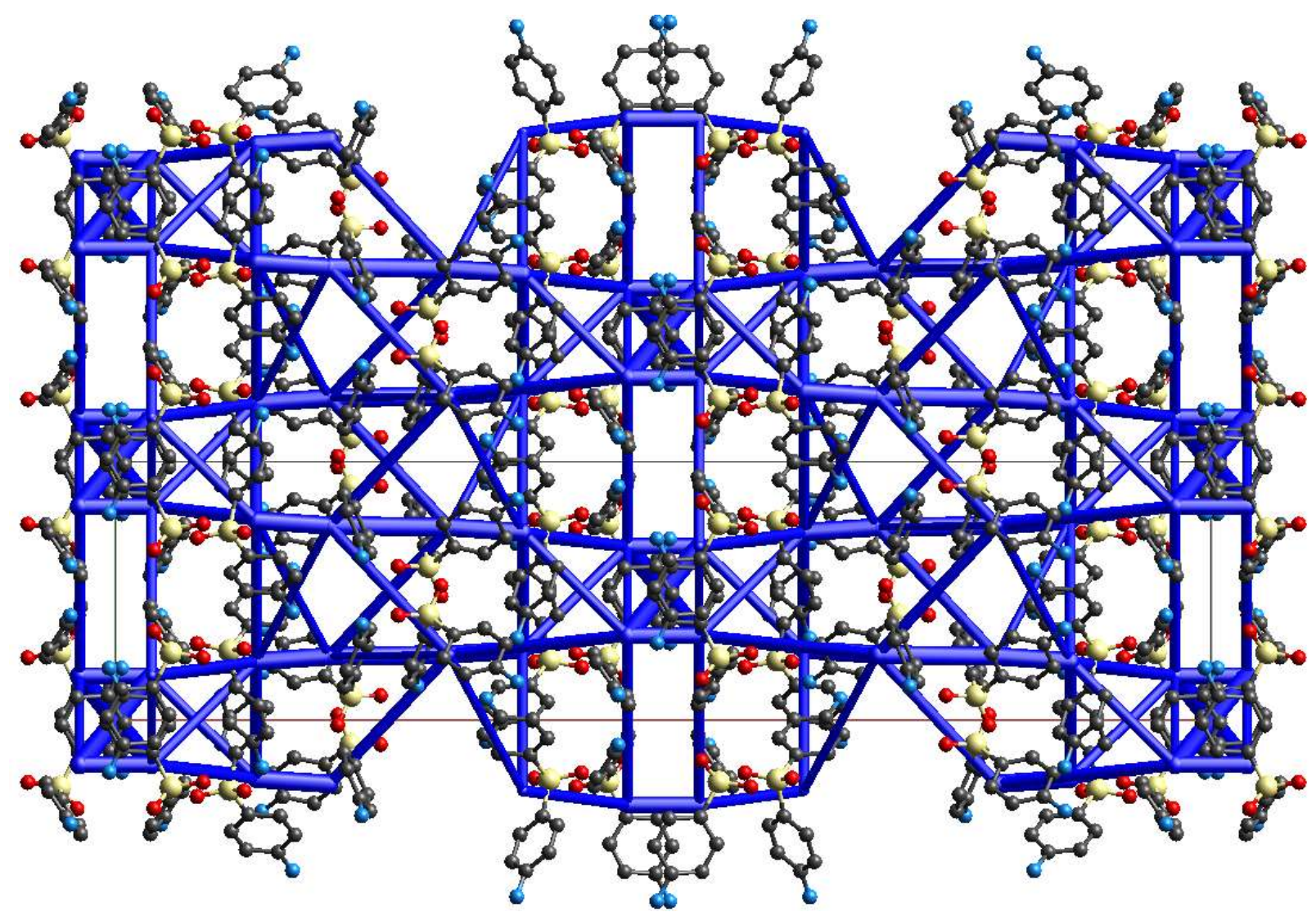

Figure S18. Energy frameworks (total energy) for Hydehy, viewed along the crystallographic $c$ axis. The energy scale factor is 80 , and pairwise interaction energies with magnitudes smaller than $20 \mathrm{~kJ} \mathrm{~mol}^{-1}$ have been omitted. 


\subsection{6_Form II}

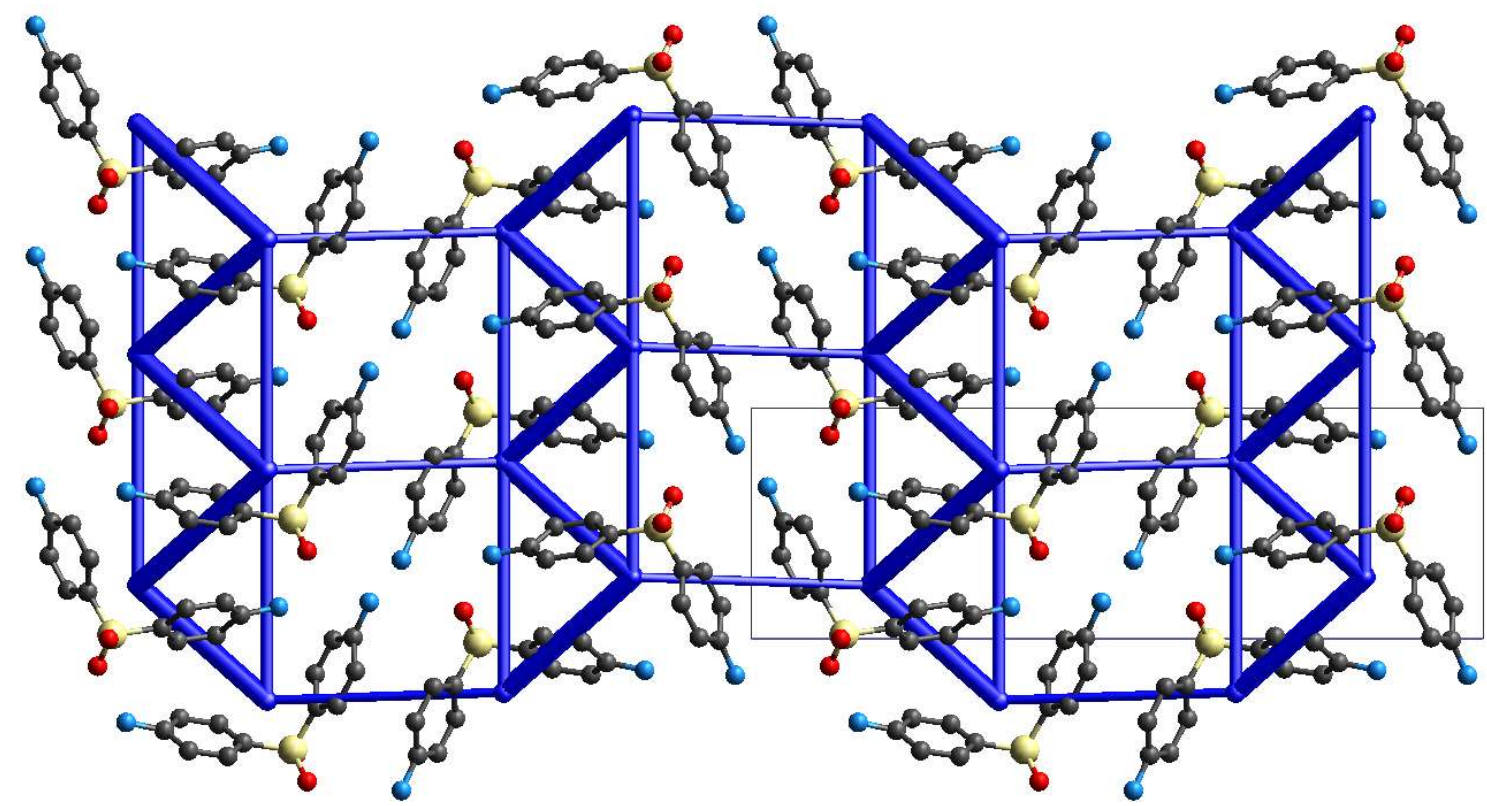

Figure S19. Energy frameworks (total energy) for Form II, viewed along the crystallographic $a$ axis. The energy scale factor is 80 , and pairwise interaction energies with magnitudes smaller than $20 \mathrm{~kJ} \mathrm{~mol}^{-1}$ have been omitted.

8.7. 07_591

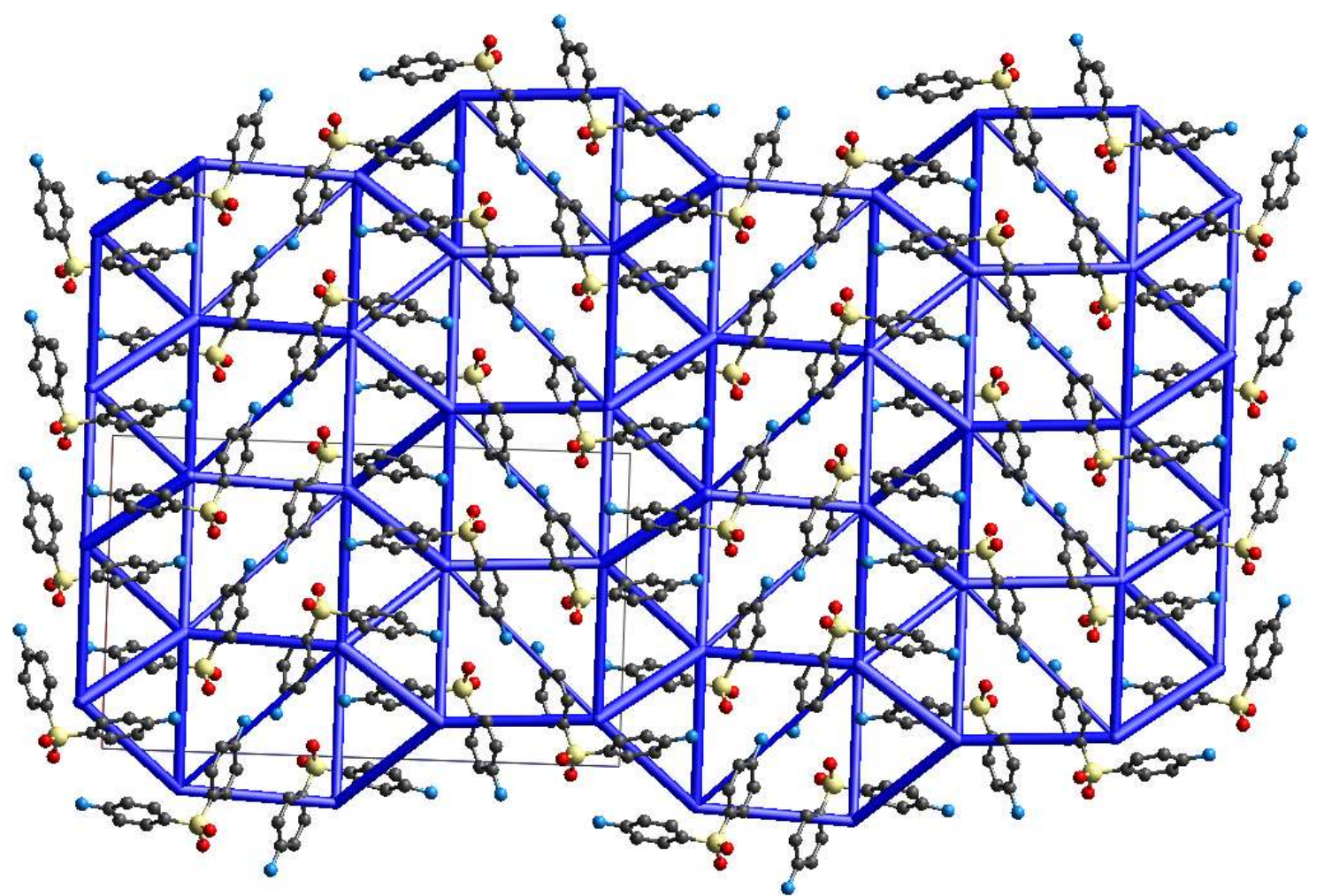

Figure S20. Energy frameworks (total energy) for 07_591, viewed along the crystallographic $b$ axis. The energy scale factor is 80 , and pairwise interaction energies with magnitudes smaller than $20 \mathrm{~kJ} \mathrm{~mol}^{-1}$ have been omitted. 


\subsection{8_Form I}

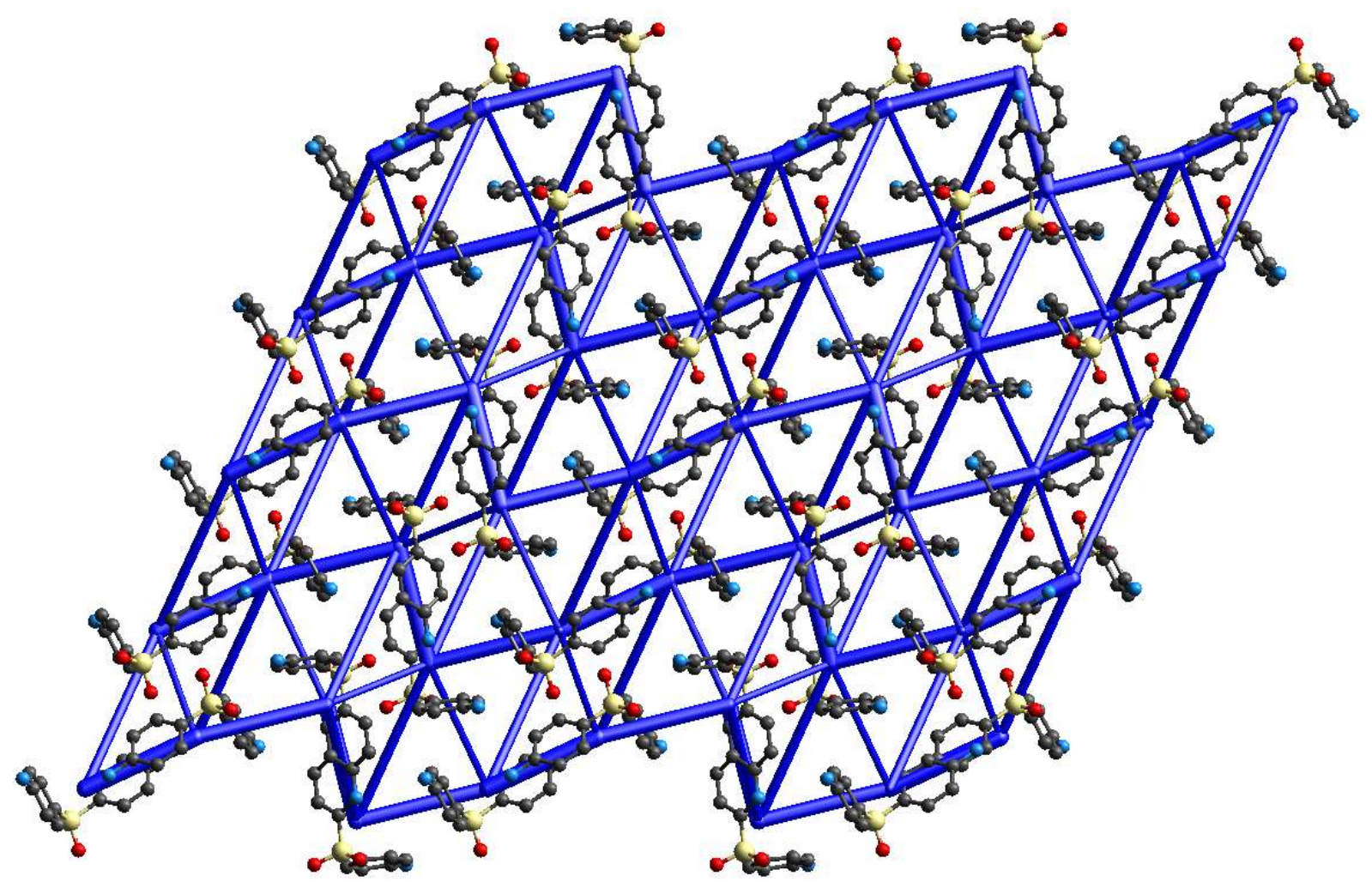

Figure S21. Energy frameworks (total energy) for Form I, viewed along the crystallographic $b$ axis. The energy scale factor is 80 , and pairwise interaction energies with magnitudes smaller than $20 \mathrm{~kJ} \mathrm{~mol}^{-1}$ have been omitted.

\subsection{9_3410}

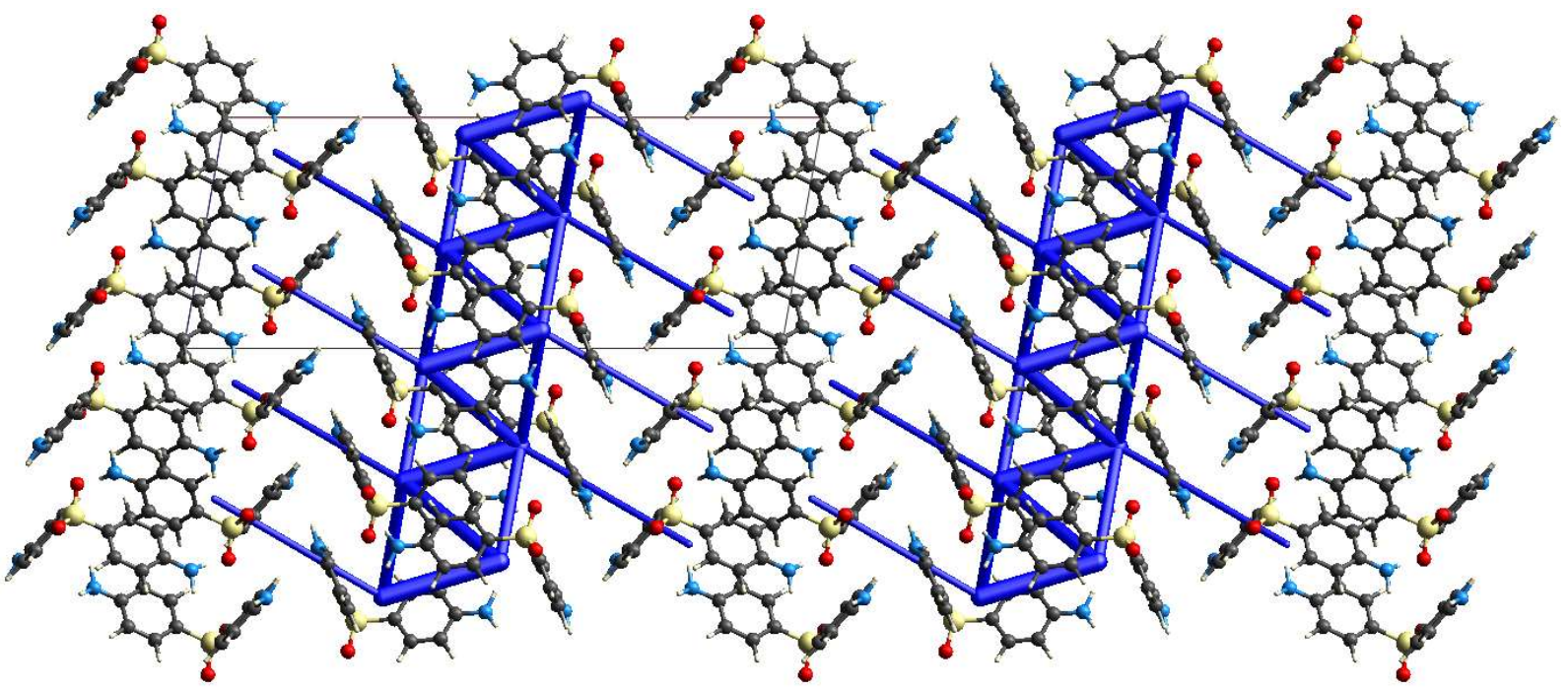

Figure S22. Energy frameworks (total energy) for 09_3410 viewed along the crystallographic $b$ axis. The energy scale factor is 80 , and pairwise interaction energies with magnitudes smaller than $20 \mathrm{~kJ} \mathrm{~mol}^{-1}$ have been omitted. 


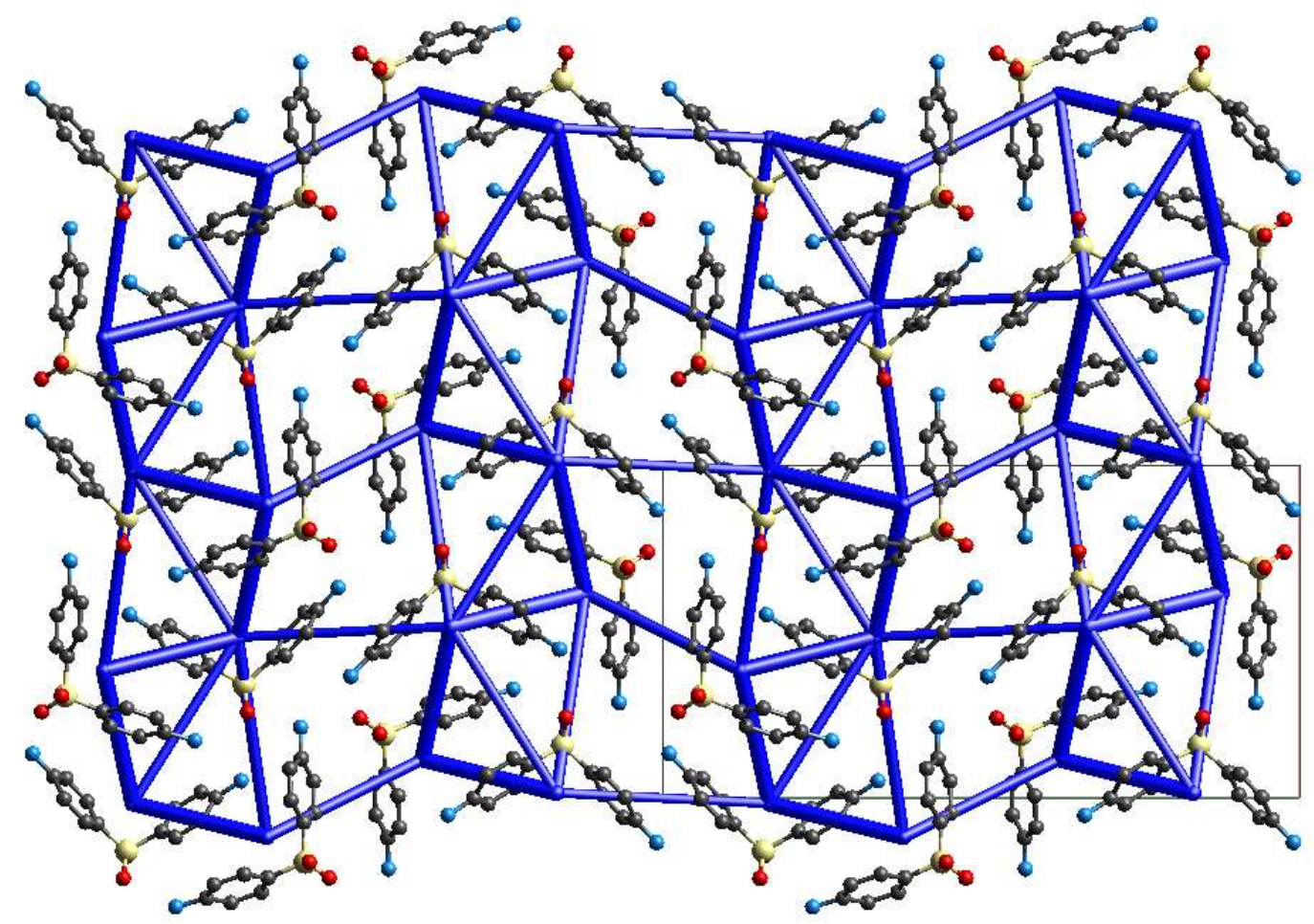

Figure S23. Energy frameworks (total energy) for 10_155, viewed along the crystallographic $c$ axis. The energy scale factor is 80 , and pairwise interaction energies with magnitudes smaller than $20 \mathrm{~kJ} \mathrm{~mol}^{-1}$ have been omitted.

8.11. 11_4478

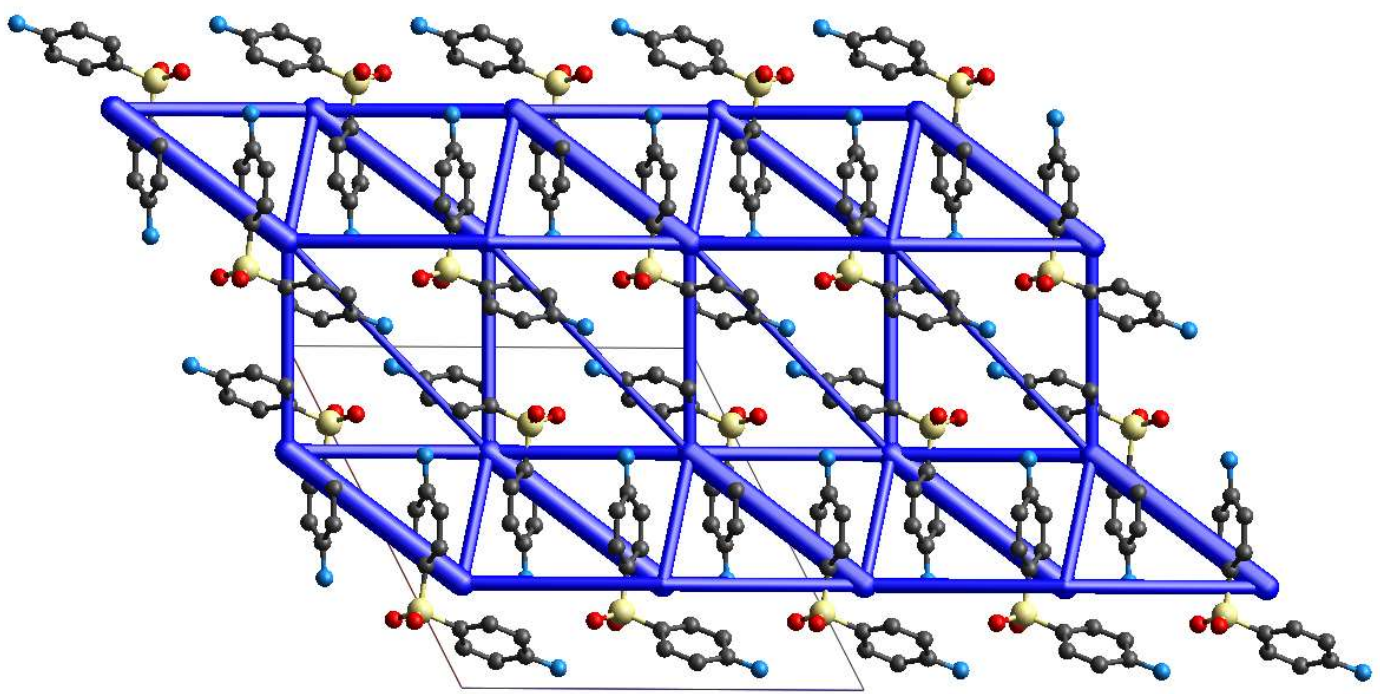

Figure S24. Energy frameworks (total energy) for 11_4478, viewed along the crystallographic $c$ axis. The energy scale factor is 80 , and pairwise interaction energies with magnitudes smaller than $20 \mathrm{~kJ} \mathrm{~mol}^{-1}$ have been omitted. 
8.12. 24_697

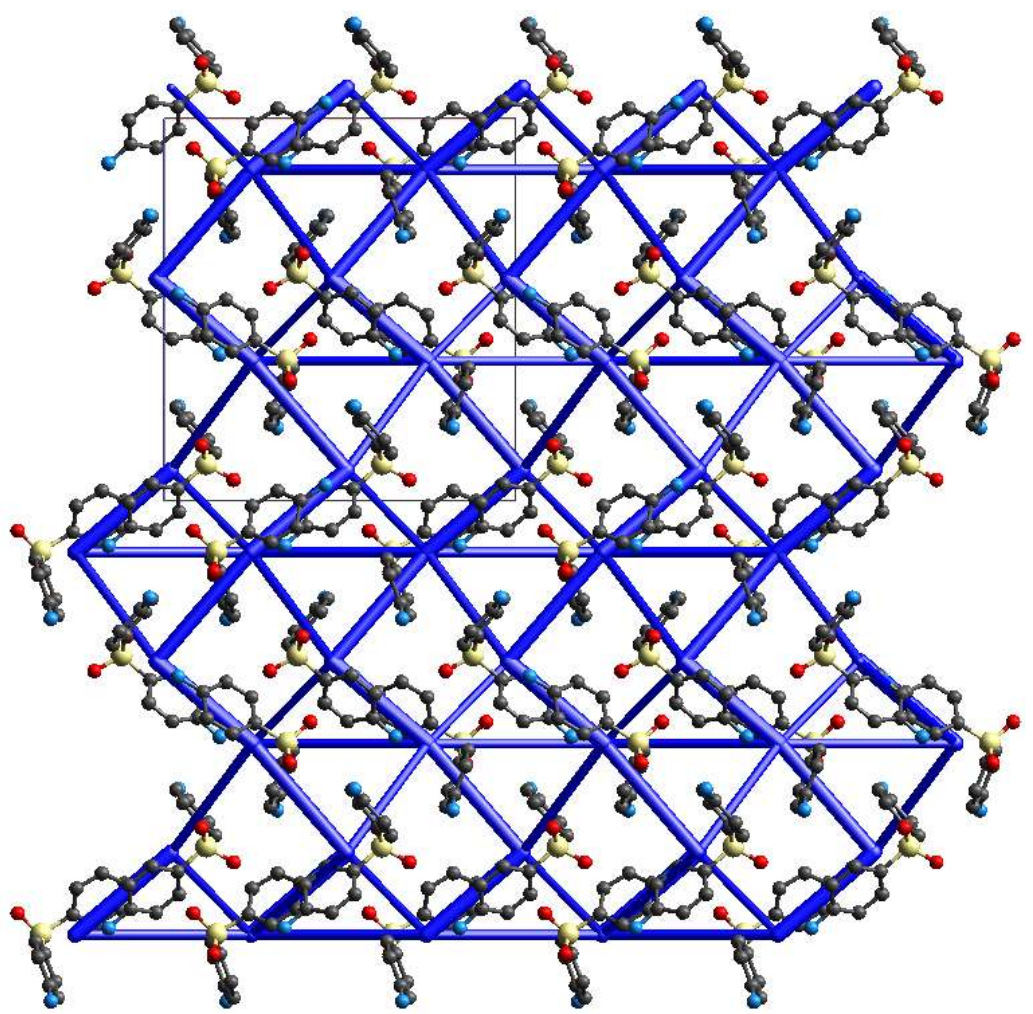

Figure S25. Energy frameworks (total energy) for 24_697, viewed along the crystallographic $b$ axis. The energy scale factor is 80 , and pairwise interaction energies with magnitudes smaller than $20 \mathrm{~kJ} \mathrm{~mol}^{-1}$ have been omitted. 


\section{DDS Structures Derived from CSP/Dehydration Modelling}

The .res files for the computationally generated structures are given.

\subsection{Form I}

TITL DDS_Form_I_RT

$\begin{array}{lllllll}\text { CELL } 1.54180 & 19.3786 & 8.3016 & 16.4960 & 90.000 & 114.533 & 90.000\end{array}$

$\begin{array}{llllllll}\text { ZERR } & 8 & 0.0000 & 0.0000 & 0.0000 & 0.000 & 0.000 & 0.000\end{array}$

LATT 1

SYMM - X, $0.50000+Y, \quad 0.50000-Z$

SFAC C H N O S

UNIT 969616168

C1 110.267590 .457120 .19961

$\begin{array}{llllll}C 2 & 1 & 0.22886 & 0.60023 & 0.19101\end{array}$

C3 10.259000 .746950 .17680

$\begin{array}{lllllllll}\text { C4 } & 1 & 0.32637 & 0.74219 & 0.16359\end{array}$

$\begin{array}{llllllllllllll}\text { C5 } & 1 & 0.36524 & 0.59879 & 0.17201\end{array}$

$\begin{array}{lllllll}\text { C6 } & 1 & 0.33678 & 0.45562 & 0.19140\end{array}$

$\begin{array}{llllllllllllll}\text { C7 } & 1 & 0.44348 & 0.27533 & 0.33153\end{array}$

$\begin{array}{llllllllllllll}C 8 & 1 & 0.50901 & 0.36967 & 0.37140\end{array}$

$\begin{array}{lllllll}\text { C9 } & 1 & 0.54998 & 0.36649 & 0.46271\end{array}$

$\begin{array}{lllllllllllllll}\text { C10 } & 1 & 0.52580 & 0.27147 & 0.51705\end{array}$

$\begin{array}{llllllllll}\text { C11 } & 1 & 0.45846 & 0.18095 & 0.47592\end{array}$

$\begin{array}{lllllllllll}\text { C12 } & 1 & 0.41799 & 0.18231 & 0.38450\end{array}$

$\begin{array}{llllllllllllll}\text { C13 } & 1 & 0.08235 & 0.81079 & 0.45233\end{array}$

$\begin{array}{lllllll}\text { C14 } & 1 & 0.04329 & 0.82623 & 0.50551\end{array}$

$\begin{array}{llllll}\text { C15 } & 1 & -0.02247 & 0.73417 & 0.48872\end{array}$

$\begin{array}{llllll}\text { C16 } & 1-0.04643 & 0.62265 & 0.41763\end{array}$

$\begin{array}{llllllll}\text { C17 } & 1-0.00683 & 0.60536 & 0.36532\end{array}$

$\begin{array}{lllllllllllll}\text { C18 } & 1 & 0.05707 & 0.70157 & 0.38128\end{array}$

$\begin{array}{llllllllllllllll}\text { C19 } & 1 & 0.15829 & 0.49575 & 0.35187\end{array}$

$\begin{array}{lllllllllllllll}\text { C20 } & 1 & 0.13244 & 0.35468 & 0.30207\end{array}$

$\begin{array}{lllllllllllllll}\text { C21 } & 1 & 0.16960 & 0.21045 & 0.33348\end{array}$

$\begin{array}{llllllllllll}\text { C22 } & 1 & 0.23305 & 0.20223 & 0.41642\end{array}$

$\begin{array}{lllllllllllll}\text { C23 } & 1 & 0.25940 & 0.34653 & 0.46510\end{array}$

$\begin{array}{lllllllllllll}\text { C24 } & 1 & 0.22223 & 0.49078 & 0.43356\end{array}$

H1 20.187780 .890070 .20762

$\begin{array}{lllllllllllllll}H 2 & 2 & 0.25366 & 0.99357 & 0.18107\end{array}$

H3 $20.609160 .34750 \quad 0.63635$

$\begin{array}{llllllllllllllllll}\text { H4 } & 2 & 0.54008 & 0.22712 & 0.64583\end{array}$

$\begin{array}{lllllll}\text { H5 } & 2 & 0.24453 & 0.34632 & 0.21373\end{array}$

$\begin{array}{llllll}\text { H6 } & 2 & 0.17516 & 0.60118 & 0.19719\end{array}$

$\begin{array}{lllllllllllll}\text { H7 } & 2 & 0.34951 & 0.85414 & 0.15105\end{array}$

$\begin{array}{llllllllll}H 8 & 2 & 0.41891 & 0.59812 & 0.16546\end{array}$

$\begin{array}{lllllllllllllll}\text { H9 } & 2 & 0.52740 & 0.44665 & 0.33065\end{array}$

$\begin{array}{lllllll}H 10 & 2 & 0.60051 & 0.44116 & 0.49364\end{array}$

$\begin{array}{lllllll}H 11 & 2 & 0.43882 & 0.10837 & 0.51708\end{array}$

$\begin{array}{llllll}H 12 & 2 & 0.36699 & 0.10924 & 0.35411\end{array}$

$\begin{array}{lllll}\text { H13 } & 2 & -0.03609 & 0.80575 & 0.60093\end{array}$

$\begin{array}{llllll}\text { H14 } & 2 & -0.10411 & 0.67253 & 0.53252\end{array}$

$\begin{array}{lllllllll}H 15 & 2 & 0.24163 & -0.04542 & 0.41667\end{array}$

$\begin{array}{lllllll}\text { H16 } & 2 & 0.30480 & 0.04859 & 0.51349\end{array}$

$\begin{array}{lllllll}H 17 & 2 & 0.13232 & 0.88477 & 0.46571\end{array}$

$\begin{array}{llllll}\mathrm{H} 18 & 2 & 0.06274 & 0.91163 & 0.56047\end{array}$

$\begin{array}{lllll}\text { H19 } & 2 & -0.09545 & 0.54654 & 0.40632\end{array}$ 
$\begin{array}{lllll}\text { H2O } & 2 & -0.02497 & 0.51646 & 0.31189\end{array}$

$\begin{array}{llllll}\mathrm{H} 21 & 2 & 0.08259 & 0.35862 & 0.23888\end{array}$

$\begin{array}{llllll}\mathrm{H} 22 & 2 & 0.14831 & 0.10018 & 0.29567\end{array}$

$\begin{array}{llllll}\mathrm{H} 23 & 2 & 0.30845 & 0.34338 & 0.52906\end{array}$

$\begin{array}{lllllll}\text { H24 } & 2 & 0.24255 & 0.60018 & 0.47298\end{array}$

N1 $3 \begin{array}{lllll}3 & 0.22311 & 0.88992 & 0.17561\end{array}$

$\begin{array}{llllll}\text { N2 } & 3 & 0.56692 & 0.26512 & 0.60725\end{array}$

$\begin{array}{lllll}\text { N3 } & 3 & -0.06250 & 0.75372 & 0.53932\end{array}$

$\begin{array}{lllll}\text { N4 } & 3 & 0.26822 & 0.05825 & 0.44791\end{array}$

$\begin{array}{llllll}01 & 4 & 0.44510 & 0.29641 & 0.17434\end{array}$

$\begin{array}{llllll}02 & 4 & 0.34075 & 0.14136 & 0.19080\end{array}$

$\begin{array}{llllll}03 & 4 & 0.05226 & 0.64814 & 0.22278\end{array}$

$\begin{array}{llllll}04 & 4 & 0.15998 & 0.80886 & 0.33122\end{array}$

$\begin{array}{llllll}\text { S1 } & 5 & 0.39200 & 0.27969 & 0.21583\end{array}$

$\begin{array}{llllll}\text { S2 } & 5 & 0.10663 & 0.67480 & 0.31467\end{array}$

END

\subsection{Form IV*}

TITL DDS_Form_VI_RT

$\begin{array}{lllllll}\text { CELL } 1.54180 & 16.4325 & 8.3091 & 17.7345 & 90.000 & 90.000 & 90.000\end{array}$

$\begin{array}{llllllll}\text { ZERR } & 8 & 0.0000 & 0.0000 & 0.0000 & 0.000 & 0.000 & 0.000\end{array}$

LATT -1

SYMM $\quad-X, \quad-Y, \quad 0.50000+Z$

SYMM $\quad 0.50000-X, \quad Y, 0.50000+Z$

SYMM $0.50000+X, \quad-Y, \quad Z$

SFAC C H N O S

UNIT $96961616 \quad 8$

C1 $110.248190 .60306 \quad 0.48489$

$\begin{array}{lllllll}C 2 & 1 & 0.11207 & 0.49308 & 0.49880\end{array}$

$\begin{array}{llllll}\text { C3 } & 1 & 0.17619 & 0.59172 & 0.52456\end{array}$

$\begin{array}{llllll}\text { C4 } & 1 & 0.12342 & 0.40548 & 0.43137\end{array}$

$\begin{array}{lllllll}\text { C5 } & 1 & 0.19568 & 0.41591 & 0.39166\end{array}$

$\begin{array}{llllll}\text { C6 } & 1 & 0.25882 & 0.51385 & 0.41842\end{array}$

$\begin{array}{llllll}\text { C7 } & 1 & 0.34005 & 0.71604 & 0.31507\end{array}$

$\begin{array}{llllll}\text { C8 } & 1 & 0.30849 & 0.71308 & 0.24176\end{array}$

$\begin{array}{lllllll}\text { C9 } & 1 & 0.30285 & 0.85364 & 0.20046\end{array}$

$\begin{array}{llllll}\text { C10 } & 1 & 0.32735 & 1.00210 & 0.23186\end{array}$

$\begin{array}{lllllll}\text { C11 } & 1 & 0.35830 & 1.00314 & 0.30613\end{array}$

$\begin{array}{lllllll}\text { C12 } & 1 & 0.36500 & 0.86221 & 0.34700\end{array}$

$\begin{array}{llllll}\text { C13 } & 1 & 0.14487 & 0.35208 & 0.12373\end{array}$

$\begin{array}{llllll}\text { C14 } & 1 & 0.12497 & 0.49725 & 0.15716\end{array}$

$\begin{array}{lllllll}\text { C15 } & 1 & 0.06650 & 0.50326 & 0.21535\end{array}$

$\begin{array}{lllllll}\text { C16 } & 1 & 0.03416 & 0.35724 & 0.24266\end{array}$

$\begin{array}{llllll}\text { C17 } & 1 & 0.05373 & 0.21241 & 0.20909\end{array}$

$\begin{array}{llllll}\mathrm{C} 18 & 1 & 0.10837 & 0.20941 & 0.14859\end{array}$

$\begin{array}{lllllll}\text { C19 } & 1 & 0.03872 & 0.04190 & 0.03110\end{array}$

$\begin{array}{llllll}\text { C20 } & 1 & -0.03338 & -0.04384 & 0.04371\end{array}$

C21 $1-0.09882-0.02434-0.00457$

C22 $1-0.093790 .08154-0.06661$

C23 $1-0.02180 \quad 0.17159-0.07653$

$\begin{array}{llllll}\text { C24 } & 1 & 0.04339 & 0.15190 & -0.02859\end{array}$

H1 $20.03141 \quad 0.563690 .58073$

H2 2 -0.01007 $0.44605 \quad 0.51066$

$\begin{array}{llllll}\text { H3 } & 2 & 0.29100 & 1.14036 & 0.14161\end{array}$

H4 $20.32880 \quad 1.248650 .21865$

H5 20.296150 .683050 .50480 

$\begin{array}{lllll}\text { H6 } & 2 & 0.16697 & 0.66370 & 0.57516\end{array}$
$\begin{array}{llllll}\text { H7 } & 2 & 0.07449 & 0.32904 & 0.41070\end{array}$
$\begin{array}{llllll}\mathrm{H} 8 & 2 & 0.20324 & 0.34712 & 0.33980\end{array}$
$\begin{array}{llllll}\text { H9 } & 2 & 0.28918 & 0.59924 & 0.21707\end{array}$
$\begin{array}{lllll}\text { H10 } & 2 & 0.27825 & 0.85222 & 0.14350\end{array}$
$\begin{array}{lllllll}H 11 & 2 & 0.37674 & 1.11701 & 0.33108\end{array}$
$\begin{array}{llllll}H 12 & 2 & 0.39050 & 0.86499 & 0.40369\end{array}$
$\begin{array}{lllllll}\text { H13 } & 2 & 0.05612 & 0.74929 & 0.21508\end{array}$
$\begin{array}{llllll}\text { H14 } & 2 & -0.01439 & 0.64786 & 0.27054\end{array}$
H15 $2-0.212280 .05066-0.10470$
H16 $2-0.15410 \quad 0.18558-0.15638$
$\begin{array}{llllll}H 17 & 2 & 0.18757 & 0.34949 & 0.07676\end{array}$
$\begin{array}{llllll}\mathrm{H} 18 & 2 & 0.15122 & 0.60924 & 0.13658\end{array}$
$\begin{array}{llllll}\text { H19 } & 2 & -0.00803 & 0.35921 & 0.28982\end{array}$
$\begin{array}{lllllll}\mathrm{H} 20 & 2 & 0.02600 & 0.10134 & 0.22919\end{array}$
H21 2 -0.03821 $-0.12608 \quad 0.09128$
H22 2 -0.15484 -0.091480 .00489$
H23 $2-0.01890 \quad 0.25988-0.12172$
$\begin{array}{llllll}\text { H24 } & 2 & 0.09821 & 0.22325 & -0.03695\end{array}$
$\begin{array}{llllll}\text { N1 } & 3 & 0.04132 & 0.48125 & 0.53899\end{array}$
$\begin{array}{llllll}\text { N2 } & 3 & 0.32323 & 1.14118 & 0.19093\end{array}$
$\begin{array}{llllll}\text { N3 } & 3 & 0.04169 & 0.64758 & 0.24525\end{array}$
N4 $3-0.15615 \quad 0.09607-0.11699$
$\begin{array}{llllll}01 & 4 & 0.35739 & 0.40416 & 0.31443\end{array}$
$\begin{array}{llllll}02 & 4 & 0.41483 & 0.56422 & 0.42161\end{array}$
O3 $4 \quad 0.10584-0.10517 \quad 0.14493$
$\begin{array}{lllllll}04 & 4 & 0.19649 & 0.04234 & 0.05493\end{array}$
S1 50.349220 .538330 .36755
$\begin{array}{llllll}\text { S2 } & 5 & 0.11921 & 0.03284 & 0.09563\end{array}$
END

\subsection{Isomorphic Dehydrate}

TITL DDS_HyDehy_OK

$\begin{array}{lllllll}\text { CELL } 1.54180 & 48.2158 & 11.3471 & 12.9500 & 90.000 & 92.639 & 90.000\end{array}$

$\begin{array}{llllllll}\text { ZERR } & 24 & 0.0000 & 0.0000 & 0.0000 & 0.000 & 0.000 & 0.000\end{array}$

LATT 7

SYMM $\quad-X, \quad Y, \quad 0.50000-Z$

SFAC C H N O S

UNIT $28828848 \quad 4824$

$\begin{array}{llllll}\text { C1 } & 1 & 0.11007 & 0.12753 & 0.71319\end{array}$

$\begin{array}{llllll}\text { C2 } & 1 & 0.13071 & 0.05626 & 0.76059\end{array}$

$\begin{array}{llllllll}\text { C3 } & 1 & 0.13810 & -0.04821 & 0.71487\end{array}$

$\begin{array}{lllllll}\text { C4 } & 1 & 0.12544 & -0.08414 & 0.61958\end{array}$

C5 $\quad \begin{array}{llllll}\text { C } & 0.10426 & -0.01223 & 0.57379\end{array}$

$\begin{array}{lllllll}\text { C6 } & 1 & 0.09680 & 0.09207 & 0.61970\end{array}$

$\begin{array}{llllll}\text { C7 } & 1 & 0.12127 & 0.36519 & 0.68057\end{array}$

$\begin{array}{llllll}\text { C8 } & 1 & 0.15037 & 0.37156 & 0.68571\end{array}$

$\begin{array}{lllllll}\text { C9 } & 1 & 0.16377 & 0.44428 & 0.61857\end{array}$

$\begin{array}{llllll}\text { C10 } & 1 & 0.14849 & 0.51092 & 0.54316\end{array}$

$\begin{array}{lllllll}\text { C11 } & 1 & 0.11924 & 0.50193 & 0.53846\end{array}$

$\begin{array}{lllllll}\text { C12 } & 1 & 0.10583 & 0.42990 & 0.60592\end{array}$

$\begin{array}{llllllll}\text { C13 } & 1 & 0.21794 & 0.17571 & 0.69509\end{array}$

$\begin{array}{llllll}\text { C14 } & 1 & 0.22976 & 0.28850 & 0.69557\end{array}$

$\begin{array}{lllllll}\text { C15 } & 1 & 0.23215 & 0.35241 & 0.78671\end{array}$

$\begin{array}{llllll}\text { C16 } & 1 & 0.22307 & 0.30460 & 0.87983\end{array}$

$\begin{array}{lllllll}\text { C17 } & 1 & 0.21121 & 0.19117 & 0.87817\end{array}$ 
$\begin{array}{llllll}\text { C18 } & 1 & 0.20860 & 0.12767 & 0.78660\end{array}$

$\begin{array}{llllll}\text { C19 } & 1 & 0.19154 & 0.17080 & 0.49629\end{array}$

$\begin{array}{llllll}\text { C20 } & 1 & 0.20048 & 0.25994 & 0.43087\end{array}$

$\begin{array}{lllllll}\text { C21 } & 1 & 0.18131 & 0.32184 & 0.36934\end{array}$

$\begin{array}{llllll}\text { C22 } & 1 & 0.15268 & 0.29619 & 0.37178\end{array}$

$\begin{array}{lllllll}\text { C23 } & 1 & 0.14412 & 0.20672 & 0.43918\end{array}$

$\begin{array}{llllllllllll}\text { C24 } & 1 & 0.16321 & 0.14551 & 0.50058\end{array}$

$\begin{array}{lllllllllll}\text { C25 } & 1 & 0.03396 & 0.12410 & 0.36383\end{array}$

$\begin{array}{llllll}\text { C26 } & 1 & 0.04884 & 0.02008 & 0.34841\end{array}$

$\begin{array}{lllllll}\text { C27 } & 1 & 0.03543 & -0.08718 & 0.35062\end{array}$

$\begin{array}{llllllllllll}\text { C28 } & 1 & 0.00691 & -0.09420 & 0.37007\end{array}$

$\begin{array}{llllll}\text { C29 } & 1 & -0.00793 & 0.01172 & 0.38220\end{array}$

$\begin{array}{lllllll}\text { C30 } & 1 & 0.00537 & 0.11911 & 0.37827\end{array}$

$\begin{array}{lllllll}\text { C31 } & 1 & 0.04911 & 0.30821 & 0.23121\end{array}$

$\begin{array}{llllllllllllll}\text { C32 } & 1 & 0.06312 & 0.24373 & 0.15776\end{array}$

$\begin{array}{llllllllllllll}\text { C33 } & 1 & 0.06097 & 0.27584 & 0.05489\end{array}$

$\begin{array}{llllll}\text { C34 } & 1 & 0.04500 & 0.37412 & 0.02261\end{array}$

$\begin{array}{llllll}\text { C35 } & 1 & 0.03182 & 0.44058 & 0.09792\end{array}$

$\begin{array}{lllllll}\text { C36 } & 1 & 0.03362 & 0.40715 & 0.20065\end{array}$

H1 $20.14811-0.237850 .60774$

H2 $20.12514-0.210330 .50260$

H3 $20.150140 .62758 \quad 0.42151$

$\begin{array}{llllll}\text { H4 } & 2 & 0.18292 & 0.58397 & 0.47509\end{array}$

$\begin{array}{llllll}\text { H5 } & 2 & 0.14112 & 0.08350 & 0.83309\end{array}$

H6 $20.15435-0.102630 .75125$

$\begin{array}{llllll}\text { H7 } & 2 & 0.09423 & -0.03803 & 0.50014\end{array}$

$\begin{array}{llllll}\text { H8 } & 2 & 0.08117 & 0.14798 & 0.58108\end{array}$

H9 $20.16258 \quad 0.318240 .74109$

$\begin{array}{llllll}\text { H10 } & 2 & 0.18636 & 0.44805 & 0.62168\end{array}$

$\begin{array}{llllll}H 11 & 2 & 0.10730 & 0.55199 & 0.48017\end{array}$

$\begin{array}{llllll}\mathrm{H} 12 & 2 & 0.08329 & 0.42312 & 0.60149\end{array}$

$\begin{array}{llllll}\text { H13 } & 2 & 0.23744 & 0.44288 & 0.97228\end{array}$

$\begin{array}{llllll}\text { H14 } & 2 & 0.22580 & 0.32234 & 1.03791\end{array}$

$\begin{array}{llllll}\mathrm{H} 15 & 2 & 0.13870 & 0.42773 & 0.27072\end{array}$

$\begin{array}{llllll}H 16 & 2 & 0.11291 & 0.33787 & 0.32023\end{array}$

$\begin{array}{llllll}\text { H17 } & 2 & 0.23742 & 0.32644 & 0.62493\end{array}$

$\begin{array}{llllll}\mathrm{H} 18 & 2 & 0.24173 & 0.43938 & 0.78686\end{array}$

$\begin{array}{llllll}\text { H19 } & 2 & 0.20418 & 0.15242 & 0.94955\end{array}$

$\begin{array}{lllll}\mathrm{H} 20 & 2 & 0.19949 & 0.03982 & 0.78574\end{array}$

$\begin{array}{llllll}\mathrm{H} 21 & 2 & 0.22239 & 0.28212 & 0.42891\end{array}$

$\begin{array}{llllll}\mathrm{H} 22 & 2 & 0.18837 & 0.39163 & 0.31890\end{array}$

$\begin{array}{llllll}\mathrm{H} 23 & 2 & 0.12214 & 0.18651 & 0.44174\end{array}$

$\begin{array}{llllll}\text { H24 } & 2 & 0.15645 & 0.07703 & 0.55244\end{array}$

$\begin{array}{llllll}\mathrm{H} 25 & 2 & 0.00626 & -0.27601 & 0.37937\end{array}$

H26 2 -0.02545 -0.208200 .39896$

$\begin{array}{llllll}\mathrm{H} 27 & 2 & 0.03717 & 0.49158 & -0.09314\end{array}$

$\begin{array}{llllll}\mathrm{H} 28 & 2 & 0.05483 & 0.36810 & -0.13124\end{array}$

$\begin{array}{llllll}\mathrm{H} 29 & 2 & 0.07091 & 0.02373 & 0.33394\end{array}$

$\begin{array}{llllll}\text { H30 } & 2 & 0.04679 & -0.16728 & 0.33454\end{array}$

$\begin{array}{llllll}\text { H31 } & 2 & -0.03002 & 0.00861 & 0.39658\end{array}$

$\begin{array}{llllll}\text { H32 } & 2 & -0.00616 & 0.20003 & 0.38928\end{array}$

$\begin{array}{llllll}\text { H33 } & 2 & 0.07559 & 0.16742 & 0.18089\end{array}$

$\begin{array}{lllllll}\text { H34 } & 2 & 0.07172 & 0.22505 & -0.00222\end{array}$

$\begin{array}{llllll}\text { H35 } & 2 & 0.01985 & 0.51818 & 0.07431\end{array}$

$\begin{array}{llllll}\text { H36 } & 2 & 0.02275 & 0.45770 & 0.25739\end{array}$

$\begin{array}{llllll}\text { N1 } & 3 & 0.13373 & -0.18388 & 0.57248\end{array}$

$\begin{array}{llllll}\text { N2 } & 3 & 0.16164 & 0.58242 & 0.47677\end{array}$ 
$\begin{array}{llllll}\text { N3 } & 3 & 0.22473 & 0.36981 & 0.97065\end{array}$

$\begin{array}{lllll}\text { N4 } & 3 & 0.13349 & 0.35303 & 0.30912\end{array}$

$\begin{array}{lllll}\text { N5 } & 3 & -0.00567 & -0.20126 & 0.37521\end{array}$

N6 $30.041350 .40359-0.08047$

$\begin{array}{llllll}01 & 4 & 0.07446 & 0.29557 & 0.75043\end{array}$

$\begin{array}{llllll}02 & 4 & 0.11740 & 0.27496 & 0.86491\end{array}$

O3 $4 \quad 0.20288-0.020240 .60299$

$\begin{array}{llllll}04 & 4 & 0.24201 & 0.09463 & 0.53233\end{array}$

$\begin{array}{lllllll}05 & 4 & 0.03499 & 0.34327 & 0.42093\end{array}$

$\begin{array}{llllll}06 & 4 & 0.07998 & 0.24151 & 0.38982\end{array}$

$\begin{array}{llllll}\text { S1 } & 5 & 0.10408 & 0.26860 & 0.76083\end{array}$

S2 $\quad \begin{array}{lllll}5 & 0.21478 & 0.09380 & 0.57859\end{array}$

$\begin{array}{llllll}\text { S3 } & 5 & 0.05070 & 0.26000 & 0.36034\end{array}$

END 


\section{References}

1. Pawley, G. S., Unit-Cell Refinement from Powder Diffraction Scans. Journal of Applied Crystallography 1981, 14 (DEC), 357-361.

2. Coelho, A. A. Topas Academic V5, Coelho Software: Brisbane, 2012.

3. Kuhnert-Brandstatter, M.; Moser, I., Polymorphism of Dapson and ethambutoldihydrochloride. Mikrochimica Acta 1979, 1 (1-2), 125-136.

4. Spek, A. L. PLATON, A Multipurpose Crystallographic Tool, Utrecht University: Utrecht, The Netherlands, 2003.

5. Chisholm, J. A.; Motherwell, S., COMPACK: a program for identifying crystal structure similarity using distances. Journal of Applied Crystallography 2005, 38, 228-231.

6. H.S. Yathirajan, P. N., B. Nagaraj, B.L. Bhaskar, D.E. Lynch, 2004.

7. Turner, M. J.; Thomas, S. P.; Shi, M. W.; Jayatilaka, D.; Spackman, M. A., Energy frameworks: insights into interaction anisotropy and the mechanical properties of molecular crystals. Chem. Commun. 2015, 51 (18), 3735-3738.

8. Turner, M. J.; Grabowsky, S.; Jayatilaka, D.; Spackman, M. A., Accurate and Efficient Model Energies for Exploring Intermolecular Interactions in Molecular Crystals. J. Phys. Chem. Lett. 2014, 5 (24), 4249-4255.

9. Mackenzie, C. F.; Spackman, P. R.; Jayatilaka, D.; Spackman, M. A., CrystalExplorer model energies and energy frameworks: extension to metal coordination compounds, organic salts, solvates and open-shell systems. IUCrJ 2017, 4 (5). 